\title{
Epichloë festucae in mutualistic association with Lolium perenne suppresses host apoplastic cysteine protease activity
}

\author{
Andrea Passarge ${ }^{1}$, Fatih Demir ${ }^{2}$, Kimberly Green ${ }^{3,4}$, Jasper R.L. Depotter ${ }^{1}$, Barry \\ Scott ${ }^{3,4}$, Pitter F. Huesgen 2,5,6, Gunther Doehlemann ${ }^{1^{*}}$, Johana C. Misas Villamil ${ }^{1^{*}}$ \\ ${ }^{1}$ Institute for Plant Sciences, University of Cologne, Cologne, Germany. \\ ${ }^{2}$ Central Institute for Engineering, Electronics and Analytics, Forschungszentrum Jülich, Jülich, \\ Germany. \\ ${ }^{3}$ School of Fundamental Sciences, Massey University, Palmerston North, New Zealand. \\ ${ }^{4}$ Bio-Protection Research Centre, Massey University, Palmerston North, NZ. \\ ${ }^{5}$ Cologne Excellence Cluster for Stress Responses in Ageing-Associated Diseases (CECAD), \\ University of Cologne, Cologne, Germany \\ ${ }^{6}$ Institute for Biochemistry, University of Cologne, Cologne, Germany \\ *Corresponding authors: jmisas@uni-koeln.de, gdoehlemann@uni-koeln.de.
}

Running title: Epichloë festucae inhibits ryegrass apoplastic cysteine proteases

Keywords: apoplast, cystatin, endophyte, papain-like cysteine protease, ryegrass. 


\section{Abstract}

Plants secrete various defence-related proteins into the apoplast, including proteases. Papain-like cysteine proteases (PLCPS) are central components of the plant immune system. To overcome plant immunity and successfully colonise their hosts, several plant pathogens secrete effector proteins inhibiting plant PLCPs. We hypothesized that not only pathogens but also mutualistic microorganisms interfere with PLCP-meditated plant defences to maintain endophytic colonisation with their hosts. Epichloë festucae forms mutualist associations with cool season grasses and produces a range of secondary metabolites that protect the host against herbivores. In this study, we performed a genome wide identification of Lolium perenne PLCPs, analysed their evolutionary relationship and classified them into nine PLCP subfamilies. Using activity-based protein profiling, we identified four active PLCPs in the apoplast of $L$. perenne leaves that are inhibited during endophyte interactions. We characterized the $L$. perenne cystatin LpCys1 for its inhibitory capacity against ryegrass PLCPs. LpCys1 inhibits LpCP2, indicating that LpCys1 might play a role in the suppression of PLCP activity during the interaction with $E$. festucae. However, since the activity of other $L$. perenne PLCPs is not sensitive to LpCys1 we propose that additional inhibitors are involved in the suppression of apoplastic PLCPs during E. festucae infection.

\section{Introduction}

1 Plants are continuously exposed to a great variety of microbes ranging from mutualists

2 to pathogens. The epidermal surface and the apoplast are primary interfaces of plant-

3 microbe interactions. The fungal endophyte Epichloë festucae forms symbiotic

4 associations with temperate Festuca and Lolium grass hosts (Leuchtmann et al., 1994).

5 Hyphae reside within the intercellular spaces between host cells and systemically 6 colonise the apoplast within the leaf sheath, leaf blade and inflorescences (May et al., 7 2008; Scott et al., 2012). In the later stages of host development, hyphae cease growing 8 and become closely attached to the host cell wall by an adhesive matrix and remain 9 metabolically active (Tan et al., 2001; Christensen and Voisey, 2007). The intercellular 10 growth of $E$. festucae within Lolium perenne is tightly regulated and the loss of key 
11 signalling components leads to a disruption in symbiosis (Tanaka et al., 2006, 2008,

12 2013; Takemoto et al., 2006, 2011; Eaton et al., 2010; Becker et al., 2015). This tight

13 association between E. festucae hyphae and the host cell wall, has been proposed to

14 facilitate endophyte-host crosstalk through the exchange of signalling molecules (Eaton

15 et al., 2011). To successfully colonise the host and survive within the apoplast, it has been proposed that E. festucae needs to suppress host defences (Schardl et al., 2004;

17 Scott et al., 2018). The apoplast is a harsh environment that harbours hydrolytic 18 enzymes such as chitinases and proteases, shown to be involved in plant defence response to microbes (Ökmen et al., 2018; Thomas and van der Hoorn, 2018). Among these, apoplastic proteases such as Papain-Like Cysteine Proteases (PLCPs) are hubs in plant immunity (Misas Villamil et al., 2016). PLCPs may release damage or microbe associated molecular patterns (DAMPs or MAMPs) as well as small signalling peptides which activate signalling cascades triggering the induction of defense responses (Ziemann et al., 2018; Paulus et al., 2020). They can also act as co-receptors and decoys to prevent pathogen colonization (Kourelis et al., 2020). Accordingly, distant related plant pathogens have evolved effectors targeting PLCPs or their regulators,

27 highlighting their importance in plant immunity (Krüger et al., 2002; Rooney et al., 2005;

28 Song et al., 2009; Kaschani et al., 2010; Bozkurt et al., 2011; Lozano-Torres et al., 2012; Clark et al., 2018; Misas Villamil et al., 2019). Pathogens can also manipulate the host to produce plant cystatins, cysteine protease inhibitors, to overcome defense responses. One example is CC9, a maize cystatin that suppresses host immunity during Ustilago maydis infection by inhibiting PLCPs (van der Linde et al., 2012a). It has been proposed that like pathogenic fungi, plant endophytic fungi secrete effector molecules to promote host colonization (Zamioudis and Pieterse, 2012; Spanu and Panstruga, 2017). However, so far only a small number of effectors from mutualistic fungi, mostly mycorrhiza, have been identified and characterized (Kloppholz et al., 2011; Plett et al.,

37 2011, 2014; Wawra et al., 2016; Perotto et al., 2018; Nostadt et al., 2020). Within the E. festucae Fl1 genome, 158 small (<300 amino acid residues in length) secreted proteinencoding genes are predicted (Hassing et al., 2019). Many of these are highly 40 expressed in planta, and differentially regulated during pathogenic E. festucae 41 associations caused by single gene deletions (Eaton et al., 2010; Schardl et al., 2013). 
42 Mutations in E. festucae genes that disrupt cell-cell fusion and other key signalling

43 pathways lead to an antagonistic interaction characterized by unregulated growth of

44 endophytic hyphae and detrimental effects on host growth (Scott et al., 2018).

45 Furthermore, key components of the activation of immune responses such as host

46 pathogenesis related (PR) and respiratory burst responses genes are down regulated

47 during mutualistic E. festucae associations (Dupont et al., 2015). Although these

48 findings have made significant contributions towards our understanding of the $E$.

49 festucae - host system, it is still largely unknown how E. festucae successfully

50 modulates host defence responses. In this study, we investigate the role of apoplastic

51 PLCPs of $L$. perenne during the interaction with E. festucae. Through computational and

52 proteomic approaches, we identified several PLCPs present and active in the leaf

53 apoplast of $L$. perenne. We show that commonly active PLCPs of uninfected plants are

54 inhibited in response to $E$. festucae interaction. We further identified an apoplastic $L$.

55 perenne - derived cystatin and analysed its inhibitory effect on apoplastic PLCPs. 


\section{Materials and methods}

Plant material

Lolium perenne cv Samson and Nicotiana benthamiana plants were grown in

61 greenhouse with long day period ( $16 \mathrm{~h}$ light) at $23^{\circ} \mathrm{C}$ and $8 \mathrm{~h}$ dark period at $20^{\circ} \mathrm{C}$ with

$6230-40 \%$ humidity. Lolium perenne infected Fl1 and CT plants were kindly provided by

63 Dr. Yvonne Becker (JKI, Julius Kühn - Institute, Braunschweig, Germany).

Strains and plasmid construction

The Golden gate modular cloning system was used to generate plasmids used for the

67 heterologous expression of PLCPs in N. benthamiana. All Oligonucleotides used for

68 PCR are listed in suppl. Table S1. To obtain pL1M-F1-LpCP2::2x35S, pL1M-F1scaffold_182|ref0000331-exonerate_est2genome-gene-0.0) and LpCathB (makerscaffold_11872|ref0015306-exonerate_est2genome-gene-0.3) were amplified from $L$. perenne cDNA via PCR. pL1M-F1-LpCP1::2x35S was amplified from LpCP1 (makerscaffold_2516|ref0039699-exonerate_est2genome-gene-0.3) leaving out the DNA sequence coding for the granulin domain. pL1M-F1-CP1Amut-nogran-mCherry::2x35S is described in Schulze Hüynck et al., 2019. The DNA fragments were ligated as previously described in Weber et al., 2011 and transformed first into E. coli Top10 competent cells and then into $A$. tumefaciens GV3101 competent cells for overexpression in N. benthamiana. For the expression of LpCys1 in E. coli, R_12141 (maker-scaffold_12141|ref0031444-exonerate_est2genome-gene-0.0) was amplified without signal peptide using L. perenne cDNA. Subsequently, the PCR product was

82 ligated with the Pvull-HF digested plasmid pRSET-GST-PP to obtain pRSET-PP83 LpCys1-noSP, which was transformed to E. coli BL21 (DE3)pLysS competent cells. All strains used are listed in suppl. Table S2.

87 Agrobacterium tumefaciens, containing the desired construct, were grown at $28^{\circ} \mathrm{C}$ in 88 liquid dYT media, supplemented with the appropriate antibiotics, until an $\mathrm{OD}_{600}$ between 
0.8 and 1.6 was reached. The cultures were diluted with $10 \mathrm{mM}$ magnesium chloride to a final $\mathrm{OD}_{600}$ of 1 . After at least one $\mathrm{h}$ incubation in darkness with $200 \mu \mathrm{M}$ acetosyringone (Sigma-Aldrich, Taufkirchen, Germany), 5 - 6 weeks old N. benthamiana

92 leaves were infiltrated with a needleless tuberculin-syringe.

Apoplastic fluid isolation from N. benthamiana and L. perenne leaves

Isolation of $N$. benthamiana apoplastic fluids was performed as described in Schulze Hüynck et al., 2019. In short, three days post Agrobacterium infiltration N. benthamiana leaves were harvested, and vacuum infiltrated with MilliQ water three times for 5 min at 60 mbar with a 2 min interval of atmospheric pressure. The leaves were surface dried, transferred to Falcon tubes and centrifuged for $20 \mathrm{~min}$ at $2000 \mathrm{~g}$ to isolate the leaves were cut ca. $3 \mathrm{~cm}$ above soil to avoid damage to the shoot apical meristem. The leaves were gently separated, and vacuum infiltrated with MilliQ water three times for 10 min at 60 mbar with a 2 min interval of atmospheric pressure and otherwise treated as N. benthamiana leaves.

\section{Activity Based Protein Profiling (ABPP)}

107 Leaf apoplastic fluid was incubated in darkness for $2 \mathrm{~h}$ at room temperature (RT) in 50 $108 \mathrm{mM}$ sodium acetate, $10 \mathrm{mM}$ DTT, DMSO and $0.2 \mu \mathrm{M}$ of activity based probe MV201 or 109 MV202 (Richau et al., 2012). Prior to labelling one set of samples were pre-incubated with $10 \mu \mathrm{M}$ or $20 \mu \mathrm{M}$ E-64 (Sigma-Aldrich, St. louis, Mississippi, USA), as negative

111 control. Labelling was stopped by the addition of $500 \mu$ lacetone, followed by protein 112 precipitation overnight at $-20^{\circ} \mathrm{C}$. The supernatant was discarded after samples were 113 centrifuged for $30 \mathrm{~min}$ at max. speed and $4^{\circ} \mathrm{C}$. The pellet was resuspended in water and $1141 \times$ SDS-loading dye. Samples were boiled for $5 \mathrm{~min}$ at $95^{\circ} \mathrm{C}$ and separated via SDS115 PAGE using 12\% or 15\% SDS gels. MV202 and MV201 labelled proteins were 116 visualised by in-gel fluorescent scanning using a rhodamine filter (Ex. 532 nm, Em. 580 $117 \mathrm{~nm}$ ) using the Chemi-Doc MP System (Bio-Rad, California, USA). Sample loading was 118 visualised via SyproRuby stain (Ex. 450 nm, Em. 610 nm; SyproRuby Invitrogen, 119 Carlsbad, California, USA), performed according to manufacturer's instructions. The 
120 Quantification of PLCP-signal via rhodamine signal strength was performed using the 121 ImageLabTM software (Bio-Rad, Hercules, CA, United States). For convolution ABPPs, 122 apoplastic fluid of mock and $E$. festucae infected $L$. perenne plants was mixed in a 1:1

123 ratio and incubated for $1 \mathrm{~h}$ at RT prior to labelling. Samples were labelled with MV202 124 as described above. After labelling, the apoplastic fluid of mock and E. festucae infected 125 L. perenne plants was mixed in a 1:1 ratio. Subsequently, samples were treated as 126 previously described. For the inhibition assays, apoplastic fluid was extracted from $N$. 127 benthamiana plants expressing L. perenne PLCPs. Prior to labelling the apoplastic fluid 128 was incubated for $30 \mathrm{~min}$ with different concentrations of recombinant LpCys1 or 129 chicken egg white cystatin (CEWC, Sigma-Aldrich, St. louis, Mississippi, USA) and Tris$130 \mathrm{HCl}(24 \mathrm{mM}$ Tris, $71 \mathrm{mM} \mathrm{NaCl}$; $\mathrm{pH}$ 7.5). As negative control one set of samples was 131 pre-incubated with $20 \mu \mathrm{M}$ E-64 (Sigma-Aldrich, St. louis, Mississippi, USA). 132 Subsequently, samples were treated as described above. PLCP pulldown using streptavidin beads

135 Leaf apoplastic fluid of three uninfected $L$. perenne plants was isolated as described 136 before. Apoplastic fluid (2.35 ml) was incubated for $4 \mathrm{~h}$ at RT in $50 \mathrm{mM}$ sodium acetate $137 \mathrm{pH}$ 6, $10 \mathrm{mM}$ DTT and 2 mM DCG-04 (Greenbaum et al., 2000). As a negative control, 138 one set of samples was incubated with an equivalent amount of DMSO instead of DCG139 04. The pulldown experiment was subsequently performed as described in Schulze 140 Hüynck et al., 2019.

142 Apoplast proteome sample preparation

143 Three biological replicates of ryegrass apoplast fluid were collected, proteins purified by 144 chloroform/methanol precipitation, cysteine residues reduced with $10 \mathrm{mM}$ DTT and 145 alkylated with $30 \mathrm{mM}$ IAA and digested with MS-grade Trypsin (Serva) for $16 \mathrm{~h}$ at $37^{\circ} \mathrm{C}$. 146 Stable isotope labelling was achieved by reductive dimethylation of peptide N-terminal 147 and Lys side chain primary amines with $20 \mathrm{mM} \mathrm{CH}_{2} \mathrm{O}$ and $20 \mathrm{mM} \mathrm{NaBH}{ }_{3} \mathrm{CN}(+28.0313$ $148 \mathrm{Da}$ ) for mock treated plants, $20 \mathrm{mM} \mathrm{CD}_{2} \mathrm{O}$ and $20 \mathrm{mM} \mathrm{NaBH}_{3} \mathrm{CN}$ (+32.0564 Da) for CT149 infected plants and $20 \mathrm{mM}^{13} \mathrm{CD}_{2} \mathrm{O}$ and $20 \mathrm{mM} \mathrm{NaBD} 3 \mathrm{CN}$ (+36.0756 Da) for Fl1 150 treatment (Boersema et al., 2009). Labelling reactions were quenched with final 100 
$151 \mathrm{mM}$ Tris- $\mathrm{HCl}$ pH 6.8 for $1 \mathrm{~h}$ at RT, pooled in a 1:1:1 ratio and subsequently separated in 152 three fractions at high $\mathrm{pH}(10 \% / 15 \% / 20 \% \mathrm{ACN}, 10 \mathrm{mM} \mathrm{NH} 4 \mathrm{OH})$ followed by a final 153 elution at acidic $\mathrm{pH}(50 \% \mathrm{ACN}, 0.1 \%$ formic acid (FA)). The fractions were evaporated

154 to dryness in a vacuum concentrator and reconstituted in $2 \%$ can, $0.1 \%$ FA prior to 155 LC/MS analysis.

Nano LC-MS/MS measurements

158 LC-MS/MS analysis was performed with an UltiMate 3000 RSCL nano-HPLC system 159 (Thermo) online coupled to an Impact II Q-TOF mass spectrometer (Bruker) via a 160 CaptiveSpray ion source boosted with acetonitrile-saturated nitrogen gas stream.

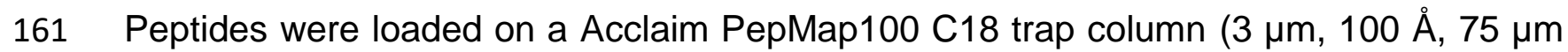
162 i.d. $x 2$ cm, Thermo) and separated on a Acclaim PepMap RSLC C18 column (2 $\mu$ m, $163100 \AA, 75 \mu \mathrm{m}$ i.d. x $50 \mathrm{~cm}$, Thermo) with a $2 \mathrm{~h}$ elution protocol that included an $80 \mathrm{~min}$ 164 separation gradient from $5 \%$ to $35 \%$ solvent $B$ (solvent $A: \mathrm{H}_{2} \mathrm{O}+0.1 \% \mathrm{FA}$, solvent $\mathrm{B}$ : 165 can, $0.1 \% \mathrm{FA}$ ) at a flow of $300 \mathrm{~nL} /$ minute at $60{ }^{\circ} \mathrm{C}$. Line-mode $\mathrm{MS}$ spectra were 166 acquired in mass range $200-1400 \mathrm{~m} / \mathrm{z}$ with a Top14 method at $4 \mathrm{~Hz}$ sampling rate for 167 MS1 spectra and an intensity-dependent acquisition rate of 5 to $20 \mathrm{~Hz}$ for MS2 spectra. 168 The capillary voltage for the CaptiveSpray ion source was 1600V. Collision energies of $1697 \mathrm{eV}$ and $9 \mathrm{eV}$ were applied in two equal steps with the ion transfer time set to 61 and $170100 \mu \mathrm{s}$, respectively, during acquisition of each MS2 spectrum.

172 Mass spectrometry data analysis

173 Peptides were identified by matching spectra against a combination of a custom 174 Epichloë festucae database (EfFl1_Proteins_Annotated_2020-05.fasta, containing 7077 175 sequences, Aug. 2018), a Lolium perenne database (lope_proteins.V1.0.fasta, 40068 176 entries, downloaded 18/03/2019, Byrne et al., 2015) and the sequences of maize and 177 Arabidopsis PLCPs (PLCPs_Ath+Maize.fasta", 52 entries) using the Andromeda search 178 engine integrated into the MaxQuant software package (version 1.6.0.16) with standard 179 settings (Tyanova et al., 2016). Carbamidomethylation of cysteine (+ $56.0214 \mathrm{Da}$ ) was 180 set as a fixed peptide modification. Oxidation of methionine (+ 15.9949 Da) and 181 acetylation of protein N-termini (+ $42.0106 \mathrm{Da})$ were set as variable modifications. For 
182 the apoplast proteome sample, triplex dimethyl isotope labelling with light $\left(\left(\mathrm{CH}_{3}\right)_{2}\right.$,

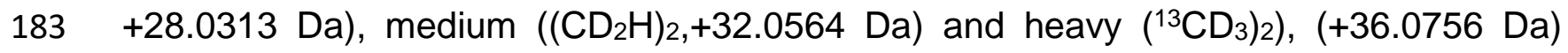

184 dimethyl label at Lys residues and peptide $\mathrm{N}$-termini was additionally considered. The 185 "requantify" option was enabled and false discovery rates (FDR) for peptide sequence 186 matches and protein identifications were set to < 0.01. Only proteins quantified in at 187 least 2 of the 3 biological replicates were used for pairwise comparisons of each of the 188 three conditions. Protein ratios were median-normalized within each replicate before 189 assessing differential expression with a moderated $t$-test using the "limma" package for $190 \mathrm{R}$ (Ritchie et al., 2015). Proteins changing at least $50 \%$ in abundance $\left(\log _{2}\right.$ fold change $191<-0.58$ or $>0.58$ ) supported by a moderated $t$-test $p$-value $<0.05$ and were considered 192 significantly changed in abundance.

Recombinant expression and purification of LpCys1

195 The Plasmid pRSET-GST-PP-LpCys1-noSP was transformed into E. coli BL21 196 (DE3)pLysS competent cells (Novagen/Merck, Darmstadt, Germany). An overnight 197 culture grown in dYT medium supplemented with $100 \mu \mathrm{g} / \mathrm{ml}$ carbenicillin and $34 \mu \mathrm{g} / \mathrm{ml}$ 198 chloramphenicol was diluted to an OD600 of 0.1 with dYT supplemented with $100 \mu \mathrm{g} / \mathrm{ml}$ 199 carbenicillin and grown at $37^{\circ} \mathrm{C}$ and $200 \mathrm{rpm}$ to an OD600 of 0.6. The LpCys1 expression 200 was induced with $1 \mathrm{mM}$ IPTG. After $4 \mathrm{~h}$ at $37^{\circ} \mathrm{C}$ and $200 \mathrm{rpm}$, the cells were harvested 201 by centrifuging for $30 \mathrm{~min}$ at $4^{\circ} \mathrm{C}$ and 6,000 rpm (JA-10, Beckman Coulter®). The cells 202 were resuspended in 1x PBS (pH 7.3) and $1 \mu$ l benzonase (Sigma-Aldrich, St. louis, 203 Mississippi, USA) and protease inhibitor mix was added. The mix was incubated for 30 $204 \mathrm{~min}$ at RT, followed by the addition of $5 \mathrm{mM}$ DTT and sonification. The insoluble cell 205 debris was removed via centrifugation for $30 \mathrm{~min}$ at $4^{\circ} \mathrm{C}$ and $20,000 \mathrm{rpm}$ (JA-25.50, 206 Beckman Coulter®). The supernatant was incubated for $1 \mathrm{~h}$ with low agitation and with $2071.2 \mathrm{ml}$ Glutathione-sepharose (GE-Healthcare, Uppsala, Sweden) that was equilibrated 208 three times with $12 \mathrm{ml}$ cold $1 \times$ PBS ( $\mathrm{pH}$ 7.3). The mix was subsequently applied to a 209 flow-through column and the column was washed three times with $12 \mathrm{ml} 1 \times \mathrm{PBS}(\mathrm{pH}$ 210 7.3) with $5 \mathrm{mM}$ DTT and once with PreScission cleavage buffer (50 mM Tris- $\mathrm{HCl} \mathrm{pH} 7.5$, $211150 \mathrm{mM} \mathrm{NaCl}, 1 \mathrm{mM}$ EDTA, $1 \mathrm{mM}$ DTT). PreScission protease mix was added and 212 incubated on the column overnight at $4{ }^{\circ} \mathrm{C}$. The flow-through was collected. The 
213 sepharose matrix was washed three times with $1.2 \mathrm{ml}$ PreScission cleavage buffer and

214 the flow-through was collected and all collected flow-through fractions were pooled. The 215 pooled protein sample was treated as describe in Mueller et al., 2013. The HiLoad®

216 16/600 Superdex® 75 pg column (GE Healthcare, Chicago, Illinois, USA) with Tris- $\mathrm{HCl}$

217 buffer (50 mM Tris-HCl, $150 \mathrm{mM} \mathrm{NaCl,} 5 \mathrm{mM}$ DTT, pH 7.5) was used for gel filtration

218 and Vivaspin 15 with 5 MWCO (Sartorius Stedim Biotech GmbH, Goettingen, Germany)

219 was used to concentrate the final protein fractions containing LpCys 1.

221 Identification and phylogenetic analysis of PLCPs and cystatins in L. perenne

222 Predicted proteins of Lolium perenne were obtained from Byrne et al., 2015 and a 223 functional prediction was carried out using InterProScan (v5.32-71.0). Subsequently it 224 was scanned for PLCP associated PFAM number PF00112 and the program SignalP 225 (v4.1) was used to identify those with an $\mathrm{N}$-terminal secretion signal. All original 226 identifiers are listed in suppl. Table S3. The PLCP phylogenetic tree was generated 227 using the identified PLCPs in L. perenne as well as 52 maize PLCP sequences from the 228 MEROPS database (B73 line), six maize PLCPs identified in the early golden bantam 229 line and 39 barley PLCP sequences (Díaz-Mendoza et al., 2014; Rawlings et al., 2018; 230 Schulze Hüynck et al., 2019). One PLCP of each PLCP subfamily from Arabidopsis 231 thaliana were also included in the tree (Richau et al., 2012), as well as four A. thaliana 232 cysteine proteases of the family C13 (legumains) $\alpha$-VPE (AEC07775.1), $\beta$-VPE 233 (OAP12170.1), Y-VPE (OAO96694.1) and $\delta$-VPE (OAP02173.1). Sequences used for 234 the phylogenetic analysis of plant PLCPs can be found in suppl. Dataset S1. For the 235 construction of the tree MAFFT (v7.407), RAxML (v8.2.12) with the PROTGAMMAWAG 236 substitution model were used (Katoh and Standley, 2013; Stamatakis, 2014). The 237 original phylogenetic tree can be found in suppl. Fig. S1. For the plant cystatins a 238 phylogenetic tree was generated by aligning the full-length protein sequences (suppl. 239 Dataset S2) of the identified apoplastic L. perenne cystatins LpCys1, LpCys4 and 240 LpCys9 as well as cystatin sequences from $\mathrm{H}$. vulgare (HvCPI), Z. mays (CC), O. sativa 241 (OC) and A. thaliana (AtCYS) using MAFFT (v7.407) (Martinez et al., 2009; Martínez et 242 al., 2012; van der Linde et al., 2012b; Katoh and Standley, 2013; Stamatakis, 2014). 243 The unrooted radial tree was generated with RAxML (v8.2.12) using the substitution 
244 model PROTGAMMAWAG (Stamatakis, 2014). 100 bootstraps were performed,

245 bootstrap values a given in the tree. The trees were visualised with FigTree (v1.4.2,

246 http://tree.bio.ed.ac.uk/software/figtree).

Results

252 Inhibition of apoplastic PLCPs has been shown to be crucial for successful infection by 253 a diversity of pathogens (Misas Villamil et al., 2016) but their role in colonization by 254 fungal endophytes is not known. In nature, different $E$. festucae strains exhibit varying degrees of host specificity and host colonisation (Scott et al., 2018). In our analysis we therefore included two E. festucae strains, Fl1 and Common Toxic (CT). Fl1 is a commonly used laboratory strain that can be artificially inoculated into the host $L$. perenne, while CT is a naturally occurring endophyte of $L$. perenne. To determine if the leaf apoplastic PLCP activity of $L$. perenne is modulated during the interaction with $E$.

260 festucae, PLCP activity was monitored using activity-based protein profiling (ABPP; Fig.

261 1). Leaf apoplastic fluid (AF) was extracted from mock and infected plants. One set of

262 plants was infected with the E. festucae strain Fl1 (sexual form), the other set of plants 263 was naturally infected seed with E. festucae var. Iolii strain CT (Common toxic, asexual 264 form). AF was labelled with the activity based probe MV201 which contains an epoxide specific E-64-based inhibitor group that covalently and irreversibly binds to the active site of PLCPs (Richau et al., 2012). A fluorescent BODIPY moiety allows the detection of MV201 labelled PLCPs. Uninfected (E-) plants showed the strongest apoplastic

268 PLCP signals at ca. $35 \mathrm{kDa}$ that were out competed showing a decrease in signal 269 intensity when the PLCP specific inhibitor E-64 was added in excess. In comparison to 270 uninfected plants, apoplastic PLCP activity in E. festucae Fl1 and CT infected plants 271 was significantly reduced (Fig. 1A). Notably, AFs from plants infected with the Fl1 strain 272 showed a stronger PLCP signal reduction than AFs from CT infected plants. 273 Furthermore, the SyproRuby loading control showed proteome differences between 274 endophyte infected samples. Despite of a high diversity in protein patterns between 
275 treatments, a distinct signal intensity for proteins at ca. $35 \mathrm{kDa}$ was observed in Fl1 276 samples, which was less intense in E- and CT samples (Fig. 1B). These findings 277 indicate that endophyte infection alters the host apoplastic proteome whereas PLCP 278 activity, a key component of plant immunity, is reduced during E. festucae interactions, 279 particularly in response to Fl1 interactions.

280 To characterize PLCP modulation in L. perenne plants it is essential to identify 281 the PLCPs in this plant species and classify them into the nine PLCP subfamilies 282 (Richau et al., 2012). Although PLCPs are crucial for many processes including plant 283 development, senescence and plant defence, the peptidase database MEROPS only 284 lists one PLCP, MER0345289, for L. perenne, belonging to the apoplastic PLCP 285 subfamily C1A (Rawlings et al., 2018). In other plant species the number of identified 286 PLCPs ranged from 63, 48, 42, 36, and 30 in maize, sorghum, barley, Arabidopsis and 287 rice, respectively (Díaz-Mendoza et al., 2014; Sekhon et al., 2019). We performed a 288 functional protease domain screen via InterProScan (www.ebi.ac.uk/interpro) using the 289 public genome annotation of Byrne et al., 2015. Subsequently, the presence of PLCP 290 related PFAM domains and IPR identifiers to determine further L. perenne PLCPS was 291 evaluated. This search identified 23 L. perenne PLCPs containing an N-terminal signal 292 peptide and a C1A protease domain (Suppl. Table S3 and Suppl. Dataset S1). To 293 classify these newly identified PLCPs into the nine PLCP subfamilies (Richau et al., 294 2012), protein sequences were compared to other plant PLCPs and phylogenetically 295 analysed. The 23 newly identified PLCPs, 58 maize PLCP sequences (Rawlings et al., 296 2018; Schulze Hüynck et al., 2019), 38 barley sequences (Díaz-Mendoza et al., 2014) 297 and one member of each PLCP subfamily of Arabidopsis (Richau et al., 2012) were 298 used to generate a phylogenetic tree using the maximum likelihood method (Fig. 2). 299 Sequences from the four Arabidopsis cysteine proteases of the family C13 (legumains) $300 \alpha-V P E, \beta-V P E, \quad \gamma-V P E$ and $\delta$-VPE were used as outgroup. This analysis showed that 301 the largest group of $L$. perenne PLCPs belong to the subfamilies THI1 (5 members), 302 SAG12 (5 members) and RD21 (4 members). The remaining seven PLCPs are 303 distributed among the XCP2, RD19A, CTB3, AALP and CEP1 subfamilies (Fig. 2). The 304 four L. perenne PLCPS R_2516 (RD21-like, subfamily I), R_4870 (AALP-like, subfamily 305 VIII), R_182 (XCP2-like, subfamily III) and R_11872 (CTB3-like, subfamily IX) were 
306 identified as closely related to the well characterized maize apoplastic PLCPs CP1, 307 CP2, XCP2 and CathB, respectively (van der Linde et al., 2012a). Therefore, we renamed these L. perenne PLCPs to LpCP1, LpCP2, LpXCP2 and LpCathB (Fig. 2).

Protein composition of the apoplast is altered during endophyte associations

The results of the ABPP experiment showing reduced activity of apoplastic PLCPs in $L$. perenne plants infected with the endophyte $E$. festucae and the large variation observed

314 in the loading controls indicate that the host apoplastic proteome is significantly altered 315 during endophyte associations. To identify changes in the proteome induced by $E$. 316 festucae infection, apoplastic fluids of infected and uninfected plants were isolated. 317 Quantitative apoplast proteome analysis using stable isotope labelling by reductive 318 dimethylation identified 1153 protein groups, of which 1092 proteins originated from $L$. 319 perenne and 86 from E. festucae (Suppl. Dataset S3). Of these, 572 (CT/mock) and 550 320 (Fl1/mock) proteins were quantified in at least 2 biological replicates for the CT/mock 321 and Fl1/mock proteomes, respectively. Among the proteins quantified in response to $322 \mathrm{CT}, 552$ belonged to L. perenne and 20 to E. festucae whereas in response to $\mathrm{Fl} 1,530$ 323 quantifiable proteins originated from L. perenne and the same 20 for E. festucae (Suppl. 324 Dataset S3). Protein abundance differed depending on the endophyte inoculation. 325 Compared to mock samples, Fl1 infected plants showed stronger changes in proteome 326 composition than CT infected plants (Fig. 3A). These differences in proteome 327 composition could be explained by the biomass variation in colonised tissue previously 328 described for $\mathrm{Fl1}$ in comparison to CT. Previous analyses have shown that Fl1 329 represents $1-2 \%$ of total biomass in infected $L$. perenne plants (Young et al., 2005) 330 whereas the biomass colonization of CT is estimated to approximately $0.2 \%$ of leaf 331 tissue in sheath (Tan et al., 2001). Notably, 51 plant proteases were identified in the 332 apoplastic proteome, including 7 aspartic proteases, 21 serine hydrolases, 9 cysteine 333 proteases and 14 proteases belonging to other classes mostly metallo-peptidases (Fig. 334 3B and Suppl. Dataset S3). Twenty-four of these plant proteases were quantified for the $335 \mathrm{Fl1} /$ mock proteome. The cysteine protease, R_8459, a SAG12-like PLCP, was 336 significantly reduced in the Fl1-infected proteome compared to mock. In contrast, two 
337 plant serine proteases, R_2759, an alpha/beta serine hydrolase of the peptidase S28

338 family and R_14255, a serine carboxypeptidase of the peptidase S10 family were 339 significantly more abundant in response to Fl1 infection (Fig. 3C, left panel). Twenty-five

340 plant apoplastic proteases were quantified in the $\mathrm{CT} /$ mock proteome although no 341 significant reduction in their abundance was observed in response to CT infection.

342 Notably, three proteases showed a significant accumulation: the same R_2759 and

343 R_14255 serine proteases also found with higher abundance in response to Fl1 and 344 R_3647, an aspartic protease with C-terminal homology to a xylanase inhibitor (Fig. 3C, 345 right panel).

347 Identification of active PLCPs in the L. perenne apoplast

Our ABPP experiment showed that uninfected $L$. perenne plants, likely resembling $L$. 350 perenne in its natural environment, maintain active PLCPs possibly to assist the 351 proteolytic activity during diverse biological processes such as development, 352 senescence, abiotic stresses and also as a response against pathogen attack. To 353 further characterize and identify active PLCPs in the apoplast of $L$. perenne a pull-down 354 of active PLCPs was performed using the activity-based probe DCG-04 (Greenbaum et 355 al., 2000). Apoplastic fluid of uninfected (E-) leaves was isolated and labelled with DCG356 04. Biotinylated proteins were affinity purified using streptavidin beads and subjected to 357 an on-bead digest (OBD) followed by mass spectrometry analysis. As a background 358 control a no-probe-control proteome was equally treated with streptavidin beads. To 359 confirm a successful affinity purification, proteins were boiled from the beads and 360 examined by western blot analysis using streptavidin-HRP antibody. Two main signals 361 between 25 and 35 kDa were observed representing PLCP labelling (Fig. 4A). MS 362 analysis identified four active PLCPs present in L. perenne apoplast: R_11872 363 (LpCathB, CTB3 subfamily IX), R_2516 (LpCP1, RD21 subfamily I), R_4870 (LpCP2, 364 AALP subfamily VIII) and R_182 (LpXCP2, XCP2 subfamily III) (Suppl. Dataset S3). 365 LpCathB and LpCp1 are the most abundant PLCPs followed by LpCP2 and LpXCP2, 366 estimated by their LFQ intensities (Fig. 4B; suppl. Dataset S3). All four active PLCPS 367 found in the apoplast of L. perenne belong to different subfamilies and share the same 
domain structures containing a signal peptide, pro-domain and mature protease with the catalytic triad Cys, His and Asn. LpCP1 additionally contains a proline-rich repeat and a granulin domain (Fig. 4B). Interestingly, the PLCP SAG12 (R_8459) which was found to

371 be significantly less abundant in the Fl1 sample (Fig. 3C) was not identified as an active

372 PLCP in the mock sample suggesting that the loss of activity observed for the Fl1

373 treated samples in the ABPP experiment correspond to the active PLCPs LpCP1,

374 LpXCP2, LpCP2 and LpCathB.

A cysteine protease inhibitor is present in the apoplast of Fl1 infected leaves

Since apoplastic PLCP activity but not the abundance of the majority of cysteine proteases was strongly reduced in response to $E$. festucae infection (Fig.1, 3C), we hypothesized that inhibitor molecules modulate PLCPs during endophytic E. festucae colonization. To test this assumption, a convolution ABPP was performed. Two apoplastic fluids $\left(\mathrm{E}\right.$ - and $\mathrm{E}_{+}$) were combined prior to labelling with the activity-based probe $(\mathrm{BL})$. If one of the apoplastic fluids $\left(\mathrm{E}_{+}\right)$contains a PLCP inhibitor in excess, the inhibitor will suppress active PLCPs present in the other apoplastic fluid (E-). To ensure that a reduction in PLCP activity is not caused by dilution, the two apoplastic fluids were also mixed after labelling (AL). Thus, monitored PLCP activity should be an average of the individual PLCP activities, when combined after labelling. A reduction of protease activity in $\mathrm{BL}$ compared to $\mathrm{AL}$ would indicate the presence of excess inhibitor in one of the apoplastic fluids (Fig. 5A;Chandrasekar et al., 2017). The convolution experiment was performed with MV202 labelled E- and E+ apoplastic fluids. E-64 pre-incubated samples served as control to ensure that observed signals were specific to the MV202 labelling and corresponded to PLCPS. The convolution ABPP revealed that combining mock (E-) and infected $\left(E_{+}\right)$apoplastic fluid before labelling $(B L)$ caused a stronger 394 reduction in PLCP activity compared to the combination after labelling (AL; Fig. 5B). 395 Notably, a strong signal at ca. 35 kDa was observed in the SyproRuby gel for the E+ 396 sample. We hypothesized that this signal could represent the stabilization of PLCPs by 397 an inhibitor. Fluorescent signal quantification of three biological experiments was 
$5 C)$, indicating that reduction in PLCP activity in response to $E$. festucae interaction is caused by an apoplastic PLCP inhibitor.

401

Mining the apoplastic proteome for potential PLCP inhibitors

To investigate if the potential apoplastic PLCP inhibitor is of endophyte origin, we screened the E. festucae Fl1 genome for orthologues of the known plant pathogen derived PLCP inhibitors Avr2 (Passalora fulva), EpiC1 and Avrblb2 (Phytophthora infestans), VAP1 (Globodera rostochiensis), Pit2 (Ustilago maydis), popP2 (Ralstonia solanacearum), SDE1 (Candidatus Liberibacter asiaticus) and Cip1 (Pseudomonas syringae pv. tomato DC3000) (Suppl. Table S4). A blastp analysis revealed two potential VAP1 orthologs, FI1_004109 and Fl1_007387 with 20.1\% and 12.04\% identity to GrVAP1, respectively. These two candidates were the only hits displaying E-values

412 with significant homology (Suppl. Table S4). In a second, unbiased, approach our

413 generated apoplastic proteome dataset was screened for the presence of Fl1 $E$.

414 festucae proteins. This approach identified $86 \mathrm{Fl} 1$ proteins of which 22 did not show

415 homology to a known PFAM domain and represent proteins with unknown function. Of

416 those 86, twenty proteins could be quantified since they were present in at least two

417 biological replicates. A functional annotation using InterProScan

418 (www.ebi.ac.uk/interpro), prediction tools for secretion signal (apoplastP 1.0, 419 http://apoplastp.csiro.au) and a prediction of a virulence function (EffectorP 1.0, 420 http://effectorp.csiro.au) was performed (Suppl. Dataset S3). From those Fl1 quantified

421 proteins only one, Fl1_003471, was predicted as an effector with apoplastic localization 422 which is more abundant in CT samples than in Fl1 samples (Suppl. Table S5 and suppl. 423 Dataset S3). From the 66 non-quantified proteins, 17 were of unknown function and 424 therefore functionally annotated as previously described (Suppl. Table S5). Fl1_002869, 425 Fl1_003333 and Fl1_005240 were predicted as putative apoplastic effectors. None of 426 the identified apoplastic E. festucae Fl1 proteins showed homology to any cysteine 427 protease inhibitor and the two orthologues of VAP1 were not found in our generated 428 proteome dataset. 
Since our analysis of the apoplastic proteome did not identify E. festucae effector candidates with significant similarity to known PLCP-inhibitors, we screened the apoplastic proteome dataset for potential PLCP inhibitors from the host plant. Seven

432 putative cysteine and serine protease inhibitors of plant origin were identified: three 433 cystatins R_12141, R_2071 and R_27228 and four serine-type peptidase inhibitors 434 (Table 1). Of these, one cystatin (R_12141) was accurately quantified in two of the three 435 experiments and did not show a significant difference in abundance between endophyte 436 (Fl1 or CT) and mock inoculated samples (Suppl. Dataset S3). A phylogenetic analysis 437 using plant cystatins from A. thaliana (Arabidopsis), H. vulgare (barley), Z. mays (maize) 438 and O. sativa (rice) as the chicken (Gallus gallus) egg white cystatin (CEWC) showed 439 three main clusters: cluster I represents only monocot cystatins, cluster II with four 440 Arabidopsis members and representative cystatins of different monocots and cluster III 441 with representative sequence homologues of AtCYS-2. R_12141 and R_2071 belonged 442 to cluster III whereas R_27228 grouped to the monocot cluster I (Fig. 6). The closest 443 homologue of $\mathrm{R} \_12141$ is $\mathrm{HvCPI}$, which is involved in barley defence against 444 Magnaporthe oryzae (Velasco-Arroyo et al., 2018). R_12141 also clustered with the $A$. 445 thaliana cystatin AtCys-1 and the maize cystatin $\mathrm{CC} 1$, and therefore this protein 446 sequence was named LpCys1. For R_2071 the closest homologue is the barley cystatin $447 \mathrm{HvCPI} 4$ and AtCys-6. Considering the close phylogeny proximity of R_27228 to the 448 maize cystatin CC9 we have named this sequence LpCys9 (Fig. 6). In summary, in our 449 proteome dataset we could not identify apoplastic proteins from E. festucae with 450 annotated function as protease inhibitors. Nevertheless, we have identified three plant 451 cystatins present in the apoplast of infected samples which could be manipulated by $E$. 452 festucae to inhibit plant PLCPs thus promoting infection and a successful colonization.

454 The L. perenne cystatin LpCys1 inhibits LpCP2

456 To further study the role of $L$. perenne cystatins in the inhibition of plant PLCPS we 457 selected LpCys1 since it was the only reliably quantified cystatin in our apoplast 458 proteome. LpCys1 is closely related to the barley cystatin HvCPI2, which has been 459 shown to strongly inhibit the barley PLCPs HvPap-6 and HvPap-10 (Martinez et al., 
460

461

462

463

464

465

466

467

468

469

470

471

472

473

474

475

476

477

478

479

480

481

482

483

484

485

486

487

488

489

490

2009). GST-tagged LpCys1 was expressed in E. coli and purified by affinity chromatography followed by gel filtration. Purified LpCys 1 was tested for its inhibitory capacity on the four apoplastic L. perenne PLCPs: LpCP1, LpCP2, LpXCP2 and LpCathB which were heterologous expressed in $N$. benthamiana leaves using Agrobacterium transient transformation. PLCP activity was determined from $N$. benthamiana apoplastic fluids containing the PLCPs using the activity based probe MV201 (Richau et al., 2012). A concentration range (0 to $4.5 \mu \mathrm{M})$ of purified LpCys1 was used for inhibition assays with the four PLCPs. Commercially available chicken cystatin (CEWC) was used as a positive control. Three controls were used in this ABPP experiment: E-64 to test for the specificity of MV201 signals, N. benthamiana expressed CP1A ${ }^{\text {mut }}$ (a catalytic inactive maize PLCP, Schulze Hüynck et al., 2019), as negative control for the PLCP background in N. benthamiana apoplastic fluids and a no-probe control (NPC), to detect unspecific fluorescent background. The activity of LpCP1 increased with low concentrations of LpCys1, reaching a maximal peak at ca. $2.5 \mu \mathrm{M}$ of incubation with the cystatin. With cystatin concentrations greater than $2.5 \mu \mathrm{M}$ the activity of LpCP1 decreases in a concentration dependent manner (Fig. 7A, B). SyproRuby staining showed a band at ca. $26 \mathrm{kDa}$, likely representing LpCP1, which increases in intensity until the addition of ca. $2.5 \mu \mathrm{M}$ LpCys1 but continues stable with increasing concentrations of the cystatin (Fig. 7A). Notably, signal quantification from four biological replicates confirmed a six-fold increase of LpCP1 activity after incubation with $2.5 \mu \mathrm{M}$ LpCys1 and further increasingly concentrations of the cystatin decreases LpCP1 activity (Fig. 7B). In contrast, CEWC showed a strong inhibition against LpCP1 already at $0.5 \mu \mathrm{M}$ (Fig. 7B and suppl. Fig. S2). In case of LpCP2, LpCys1 showed a concentration dependent inhibitory effect, resulting in complete inactivation of the protease already at $3 \mu \mathrm{M}$ LpCys1 (Fig. 7C). Inhibition of LpCP2 was also observed by CEWC, although with a much weaker inhibitory capacity than LpCys1 (Fig. 7D \& Suppl. Fig. S2). LpXCP2 was poorly inhibited by both, LpCys1 and CC, and ca. $50 \%$ of the inhibition was reached with $4.5 \mu \mathrm{M}$, the maximum tested concentration for both inhibitors, suggesting that LpCys1 has a poor affinity towards LpXCP2 (Fig. 7E-F and Suppl. Fig. S2). Finally, LpCys1 did not inhibit LpCathB although CEWC showed a concentration dependent inhibitory effect suggesting that LpCathB might not be a target 
491 for LpCys1 (Fig. 7G-H \& Suppl. Fig. S2). These results suggest that LpCys1 has a 492 stronger inhibitory capacity against LpCP2 than towards other PLCPs. Thus, LpCys1 is

493 a potent and specific inhibitor of LpCP2 that might contribute to the general reduction of 494 apoplastic PLCP activity during E. festucae colonization. However, since only one out of 495 four active proteases is sensitive to this cystatin, it is likely that additional inhibitors are 496 involved in PLCP inactivation during this fungal interaction.

\section{Discussion}

In this study we have shown that apoplastic papain-like cysteine proteases are inhibited 501 during endophytic colonisation of the L. perenne ryegrass, similar to pathogen 502 colonisation. We identified the PLCPs LpCP1, LpCP2, LpXCP2 and LpCathB being 503 active in the apoplast of uninfected L. perenne plants. Interestingly, these PLCPS 504 classify into different PLCP subfamilies, known to be hubs in plant immunity (Misas 505 Villamil et al., 2016). The most abundant of the four active apoplastic PLCPs in 506 uninfected L. perenne leaves was LpCathB. In both, N. benthamiana and $A$. thaliana 507 homologues of LpCathB are involved in the hypersensitive response triggered by 508 bacterial avirulent pathogens (Gilroy et al., 2007; McLellan et al., 2009; Ge et al., 2016) 509 although it might not function as a universal regulator of the hypersensitive response 510 (Thomas and van der Hoorn, 2018). The second most abundant active apoplastic PLCP 511 was LpCP1, followed by LpCP2 and LpXCP2. All three proteases have orthologues in 512 maize which were found to be activated in the apoplast after salicylic acid treatment and 513 are involved in the defence response against the biotrophic fungus $U$. maydis (van der 514 Linde et al., 2012a; Mueller et al., 2013). Maize CP1 and CP2 apoplastic proteases are 515 targeted and inhibited by the $U$. maydis effector Pit2 which acts as a substrate mimic 516 molecule to achieve a successful inhibition (Mueller et al., 2013; Misas Villamil et al., 517 2019). LpCP1 belongs to the RD21-like subfamily (I) and contains a granulin domain, 518 which is exclusively found in members of subfamily I or IV (Richau et al., 2012; Misas 519 Villamil et al., 2016). Members of this subfamily have been described to play a crucial 520 role during pathogen attack. The tomato C14 protease is inhibited during Phytophthora 521 infestans infection by the cystatin-like effector proteins EpiC1 and EpiC2B (Kaschani et 
522 al., 2010) and by the chagasin-like Cip1 inhibitor during Pseudomonas infection (Shindo 523 et al., 2016). Additionally, the RxLR effector of $P$. infestans targets $\mathrm{C} 14$ to prevent its 524 secretion into the apoplast (Bozkurt et al., 2011). Moreover, in barley, which is 525 phylogenetically closely related to ryegrass, HvPap-6 accumulated after Magnaporthe 526 oryzae treatment, particularly at late stages of infection and after infestation with the 527 mite Tetranychus urticae where mostly the pre-mature form of HvPap-6 accumulated 528 (Diaz-Mendoza et al., 2017). LpCP2 belongs to the AALP-like subfamily (VIII) and like 529 its closest homologue in barley, the thiol protease aleurain Hv-Pap12, LpCP2 is also 530 present and active in barley leaf extracts (Frank et al., 2019). Similar to LpCP1 531 subfamily members, LpCP2 orthologues play a role in defence against different 532 pathogens. Silencing of CYP1/2 in $N$ benthamiana increases susceptibility against 533 Colletotrichum destructivum (Hao et al., 2006). In maize, CP1 and CP2 have been 534 shown to function in the release of Zip1, a peptide signalling molecule that activates 535 salicylic acid immune responses (Ziemann et al., 2018). Together, this body of evidence 536 indicate that members related to these PLCP subfamilies need to be shut-down by 537 pathogens to avoid activation of plant immunity.

538 Do endophytes need to apply similar strategies on modulation of PLCPS as 539 pathogens? In E. festucae Fl1 and CT infected L. perenne plants the abundance of 540 PLCPs did not significantly differ from mock plants, although PLCP activity was almost 541 fully diminished, indicating that the reduction in PLCP activity is caused via inhibition 542 rather than protein degradation. Based on the results presented here, we speculate two 543 ways of inhibition: the putative inhibitor could be of plant origin, manipulated by $E$. 544 festucae to achieve a successful colonization, or of fungal origin, a secreted effector 545 molecule. An example of an inhibitor of plant origin is the cystatin CC9 which was 546 identified as an important compatibility factor in the maize - $U$. maydis interaction. Upon $547 U$. maydis infection cc9 expression is induced and CC9 suppresses plant immunity via 548 PLCP inhibition, thus enabling U. maydis colonization (van der Linde et al., 2012a). CC9 549 inhibits all apoplastic PLCPs and is required for early stages of $U$. maydis colonization 550 since at later stages of infection the Pit2 inhibitor likely takes over as a more specific 551 inhibitor of the apoplastic proteases CP1 and CP2 (van der Linde et al., 2012a; Misas 552 Villamil et al., 2019). Interestingly, the mechanism of inhibition of CP1 and CP2 by $U$. 
553 maydis Pit2 resembles the "activation - inhibition" of LpCys1 towards LpCP1 and 554 LpCP2. Whilst in maize CP2 is inhibited by Pit2, CP1 is first stabilized leading to an increased CP1 activity and eventually, at higher concentrations of Pit2, to inhibition

556 (Misas Villamil et al., 2019). In case of the L. perenne - E. festucae interaction, LpCys1 557 did not efficiently inhibit LpCP1 but rather activates it, contrary to LpCP2 where LpCys1 558 achieves a full inhibition. These results suggest that LpCys1 is likely stabilizing LpCP1 559 until the batch of available zymogen has been consumed and the inhibition can then 560 take place in a concentration dependent manner.

LpXCP2 and LpCathB are not inhibited by the cystatin LpCys1. These results 562 indicate that another inhibitor besides LpCys1 is involved in the PLCP inhibition in 563 response to E. festucae interaction. CC9 is not the closest orthologue of LpCys1 but of 564 LpCys9 which might therefore represent an interesting candidate for ryegrass PLCP 565 inhibition. If LpCys9 expression is similarly to CC9, one could speculate that it might be 566 transiently activated at early stages of infection. In this study we did not examine early 567 stages of infection which might be reflected in the innermost leaf blade of the 568 pseudostem tissue (Schmid et al., 2016), nevertheless a strong PLCP inhibition was 569 observed during endophytic interactions indicating the production of a PLCP inhibitor 570 also in a long term systemic host colonization. These findings confirm that endophyte 571 infections of $L$. perenne lead to major alterations of the host metabolism, development 572 and apoplastic proteome (Scott et al., 2018; Green et al., 2020).

573 The presence of LpCys1 in the apoplast could have an alternative function 574 unrelated to the inhibition of PLCPs. In barley, the HvCPI-2 cystatin, the closest 575 orthologue to LpCys1, showed a strong fungicide effect against Botrytis cinerea and 576 Fusarium oxysporum mycelia (Abraham et al., 2006), suggesting that the presence of 577 LpCys1 could be part of the plant immune response against E. festucae, rather than 578 LpCys1 being manipulated by E. festucae to facilitate PLCP inhibition. Indeed, one of 579 the most highly expressed fungal genes in planta is a chitinase (Eaton et al., 2010), 580 suggesting host defence responses are activated and E. festucae evades plant 581 immunity by altering or masking the chitin composition of hyphae in planta (Becker et 582 al., 2016). 
Based on our results it is possible that the inhibition of ryegrass PLCPs during mutualistic interactions is the result of a cooperative effect of plant cystatins and a secreted effector from $E$. festucae. We have identified four putative effector candidates present in our apoplast analysis and two VAP-1 orthologues that could potentially inhibit L. perenne PLCPs. Both E. festucae VAP1- like proteins match to an allergen V5/SCP domain containing protein of Claviceps purpurea and Moelleriella libera based on the uniprot database (www.uniprot.org). Fl1_004109 also has a 'hit' with a basic form of pathogenesis protein 1 of Pochonia clamydospora. Notably, all best 'hits' correspond to proteins restricted to the order Hypocreales (Claviceps spp., Metarhizium spp.,

592 Pochonia spp., Moelleriella spp., Ustilaginoiea spp.) suggesting that both E. festucae 593 identified VAP1 orthologues might be ubiquitous proteins of the family Clavicipitaceae 594 and not specific effectors from E. festucae. Notably, common features can be found for 595 cystatins such as the Q-V-G motif (Gln-Xaa-Val-Xaa-Gly), a Pro -Trp or Leu -Trp 596 dipeptide motif in the C-terminal region and a conserved Gly residue in the $\mathrm{N}$-terminal 597 region (Benchabane et al., 2010). These common features are not found in pathogen 598 derived PLCP inhibitors suggesting that pathogens evolve independent strategies to 599 suppress protease activity making challenging bioinformatic searches of this type of 600 effector molecules. Whether the new potential $E$. festucae effector candidates contribute 601 to the full inhibition during Fl1 interactions remains to be elucidated.

In summary, we have shown that during the L. perenne-E. festucae interaction, 603 microbial endophytes modulate essential components of the plant immune system, 604 similar to pathogenic interactions. In this case, the inhibition of apoplastic cysteine 605 proteases might be essential and required for $E$. festucae to maintain a mutualistic 606 interaction with its host. 


\section{Figure legends}

610 Fig. 1. Activity of apoplastic PLCPs in Epichloë festucae infected Lolium perenne

611 leaves.

612 Fig. 2. Phylogeny and subfamily classification of L. perenne PLCPS.

613 Fig. 3. Apoplast proteome analysis of Fl1 and CT infected and mock-treated leaves.

614 Fig. 4. Identification of L. perenne active apoplastic PLCPS.

615 Fig. 5. An apoplastic PLCP inhibitor is present in E. festucae infected plants.

616 Fig. 6. Phylogentic analysis of identified cystatins from L. perenne.

617 Fig. 7. Inhibitory activity of LpCys1 on overexpressed ryegrass PLCPs.

\section{Supplementary data}

620 Table S1: oligonucleotides

621 Table S2: strains

622 Table S3: original identifiers of L. perenne

623 Table S4: Functional annotation of "unknown" Fl1 apoplastic proteins

624 Table S5: screen of PLCP-inhibitor orthologs in E. festucae Fl1 strain

625 Fig. S1: Phylogenetic tree of L. perenne PLCPS

626 Fig. S2: Concentration range of the inhibition of L. perenne PLCPs by CEWC

627 Dataset S1: sequences used for PLCP phylogenetic analysis

628 Dataset S2: sequences used for cystatin phylogenetic analysis

629 Dataset S3: apoplastic proteomics, annotation and functional analysis

\section{Acknowledgements}

632 We would like to thank Yvonne Becker for providing the infected plants, for reading the 633 manuscript and for giving us helpful comments and suggestions. We also thank Hermen 634 Overkleeft (Leiden University) for kindly providing us with the ABPs. Many thanks to Ute 635 Meyer for fruitful discussions and technical support. Also, thanks to David Winter 636 (Massey University) for providing the E. festucae Fl1 protein dataset. Work in the Scott 
637 laboratory was supported by a grant (RM19009) from the Tertiary Education

638 Commission to the Bio-Protection Research Centre, B.S. was supported by an 639 Alexander von Humboldt Research Award.

\section{Author contributions}

641 A.P. and J.C.M.V. wrote the manuscript with input from all authors. G.D., J.C.M.V., B.S.,

642 P.F.H and A.P. designed the experiments. A.P., K.G, and J.C.M.V. performed the 643 biochemical characterization of ryegrass PLCPS. J.R.L.D and A.P. made the 644 bioinformatic and phylogenetic analyses of plant PLCPs and cystatins. F.D. and P.F.H. 645 performed and analysed the mass spectrometry experiments.

\section{Data availability statement}

647 All data supporting the findings of this study are available within the paper and within its 648 supplementary materials published online. All MS-based proteomics data have been 649 deposited to the ProteomeXchange Consortium via the PRIDE (Perez-Riverol et al., 650 2019) partner repository with the identifiers PXD022007 (reviewer login: 651 reviewer pxd022007@ebi.ac.uk, password: ECUnxNOH) for the ABPP dataset and 652 PXD022009 (reviewer login: reviewer_pxd022009@ebi.ac.uk, password: zQhfJ1pi) for 653 the apoplast proteome dataset. 


\section{References}

655

656

657

658

659

660

661

662

663

664

665

666

667

668

669

670

671

672

673

674

675

676

677

678

679

680

681

682

683

684

685

Abraham Z, Martinez M, Carbonero P, Diaz I. 2006. Structural and functional diversity within the cystatin gene family of Hordeum vulgare. Journal of Experimental Botany 57, 4245-4255.

Becker M, Becker Y, Green K, Scott B. 2016. The endophytic symbiont Epichloë festucae establishes an epiphyllous net on the surface of Lolium perenne leaves by development of an expressorium, an appressorium-like leaf exit structure. The New Phytologist 211, 240-254.

Becker Y, Eaton CJ, Brasell E, May KJ, Becker M, Hassing B, Cartwright GM, Reinhold L, Scott B. 2015. The Fungal Cell-Wall Integrity MAPK Cascade Is Crucial for Hyphal Network Formation and Maintenance of Restrictive Growth of Epichloë festucae in Symbiosis With Lolium perenne. Molecular plant-microbe interactions: MPMI 28, 69-85.

Benchabane M, Schlüter U, Vorster J, Goulet M-C, Michaud D. 2010. Plant cystatins. Biochimie 92, 1657-1666.

Boersema PJ, Raijmakers R, Lemeer S, Mohammed S, Heck AJR. 2009. Multiplex peptide stable isotope dimethyl labeling for quantitative proteomics. Nature Protocols 4, 484-494.

Bozkurt TO, Schornack S, Win J, et al. 2011. Phytophthora infestans effector AVRblb2 prevents secretion of a plant immune protease at the haustorial interface. Proceedings of the National Academy of Sciences 108, 20832-20837.

Byrne SL, Nagy I, Pfeifer M, et al. 2015. A synteny-based draft genome sequence of the forage grass Lolium perenne. The Plant Journal: For Cell and Molecular Biology 84, 816-826.

Chandrasekar B, Hong TN, van der Hoorn RAL. 2017. Inhibitor Discovery by Convolution ABPP. Methods in Molecular Biology (Clifton, N.J.) 1491, 47-56.

Clark K, Franco JY, Schwizer S, et al. 2018. An effector from the Huanglongbing-associated pathogen targets citrus proteases. Nature Communications 9, 1718.

Díaz-Mendoza M, Velasco-Arroyo B, González-Melendi P, Martínez M, Díaz I. 2014. C1A cysteine protease-cystatin interactions in leaf senescence. Journal of Experimental Botany 65, 3825-3833.

Diaz-Mendoza M, Velasco-Arroyo B, Santamaria ME, Diaz I, Martinez M. 2017. HvPap-1 C1A Protease Participates Differentially in the Barley Response to a Pathogen and an Herbivore. Frontiers in Plant Science 8.

Dupont P-Y, Eaton CJ, Wargent JJ, Fechtner S, Solomon P, Schmid J, Day RC, Scott B, Cox MP. 2015. Fungal endophyte infection of ryegrass reprograms host metabolism and alters development. New Phytologist 208, 1227-1240. 

signaling in a fungal-grass symbiosis leads to pathogenesis. Plant Physiology 153, 1780-1794.

Eaton CJ, Cox MP, Scott B. 2011. What triggers grass endophytes to switch from mutualism to pathogenism? Plant Science 180, 190-195.

690 Frank S, Hollmann J, Mulisch M, Matros A, Carrión CC, Mock H-P, Hensel G, Krupinska K. 2019. Barley cysteine protease PAP14 plays a role in degradation of chloroplast proteins. Journal of Experimental Botany 70, 6057-6069. 2016. Inhibition of cathepsin B by caspase-3 inhibitors blocks programmed cell death in Arabidopsis. Cell Death and Differentiation 23, 1493-1501.

696 Gilroy EM, Hein I, van der Hoorn R, et al. 2007. Involvement of cathepsin B in the plant disease resistance hypersensitive response. The Plant Journal: For Cell and Molecular Biology 52, 1-13. 2020. Lolium perenne apoplast metabolomics for identification of novel metabolites produced by the symbiotic fungus Epichloë festucae. New Phytologist 227, 559-571.

Greenbaum D, Medzihradszky KF, Burlingame A, Bogyo M. 2000. Epoxide electrophiles as activity-dependent cysteine protease profiling and discovery tools. Chemistry \& Biology 7, 569703581.

Hao L, Hsiang T, Goodwin PH. 2006. Role of two cysteine proteinases in the susceptible response of Nicotiana benthamiana to Colletotrichum destructivum and the hypersensitive response to Pseudomonas syringae pv. tomato. Plant Science 170, 1001-1009. festucae small secreted proteins in the interaction with Lolium perenne. PLoS ONE 14. der Hoorn RAL. 2010. An Effector-Targeted Protease Contributes to Defense against Physiology 154, 1794-1804.

Katoh K, Standley DM. 2013. MAFFT multiple sequence alignment software version 7:

715 Kloppholz S, Kuhn H, Requena N. 2011. A Secreted Fungal Effector of Glomus intraradices 716 Promotes Symbiotic Biotrophy. Current Biology 21, 1204-1209.

717 Kourelis J, Malik S, Mattinson O, Krauter S, Kahlon PS, Paulus JK, van der Hoorn RAL. 2020.

718 Evolution of a guarded decoy protease and its receptor in solanaceous plants. Nature

719 Communications 11, 4393. 
720 Krüger J, Thomas CM, Golstein C, Dixon MS, Smoker M, Tang S, Mulder L, Jones JDG. 2002. A

721 Tomato Cysteine Protease Required for Cf-2-Dependent Disease Resistance and Suppression of

722 Autonecrosis. Science 296, 744-747.

723 Leuchtmann A, Schardl CL, Siegel MR. 1994. Sexual compatibility and taxonomy of a new

724 species of Epichloë symbiotic with fine fescue grasses. Mycologia 86, 802-812.

van der Linde K, Hemetsberger C, Kastner C, Kaschani F, van der Hoorn RAL, Kumlehn J, Doehlemann G. 2012a. A maize cystatin suppresses host immunity by inhibiting apoplastic cysteine proteases. The Plant Cell 24, 1285-1300.

van der Linde K, Mueller AN, Hemetsberger C, Kashani F, van der Hoorn RAL, Doehlemann G. $2012 b$. The maize cystatin CC9 interacts with apoplastic cysteine proteases. Plant Signaling \&

730 Behavior 7, 1397-1401.

Lozano-Torres JL, Wilbers RHP, Gawronski P, et al. 2012. Dual disease resistance mediated by the immune receptor $\mathrm{Cf}-2$ in tomato requires a common virulence target of a fungus and a nematode. Proceedings of the National Academy of Sciences of the United States of America

734 109, 10119-10124.

M. J., Christensen, C. R., Voisey. 2007. The biology of the endophyte/grass partnership. New Zealand Grassland Association: Endophyte Symposium, 123-133.

Martinez M, Cambra I, Carrillo L, Diaz-Mendoza M, Diaz I. 2009. Characterization of the entire cystatin gene family in barley and their target cathepsin L-like cysteine-proteases, partners in the hordein mobilization during seed germination. Plant Physiology 151, 1531-1545.

740 Martínez M, Cambra I, González-Melendi P, Santamaría ME, Díaz I. 2012. C1A cysteine-

741 proteases and their inhibitors in plants. Physiologia Plantarum 145, 85-94.

742 May KJ, Bryant MK, Zhang X, Ambrose B, Scott B. 2008. Patterns of Expression of a Lolitrem 743 Biosynthetic Gene in the Epichloë festucae-Perennial Ryegrass Symbiosis. Molecular Plant-

744 Microbe Interactions 21, 188-197.

745 McLellan H, Gilroy EM, Yun B-W, Birch PRJ, Loake GJ. 2009. Functional redundancy in the 746 Arabidopsis Cathepsin B gene family contributes to basal defence, the hypersensitive response 747 and senescence. New Phytologist 183, 408-418.

748 Misas Villamil JC, Mueller AN, Demir F, et al. 2019. A fungal substrate mimicking molecule 749 suppresses plant immunity via an inter-kingdom conserved motif. Nature Communications 10, 7501576.

751 Misas-Villamil JC, van der Hoorn RAL, Doehlemann G. 2016. Papain-like cysteine proteases as 752 hubs in plant immunity. The New Phytologist 212, 902-907. 
Mueller AN, Ziemann S, Treitschke S, Aßmann D, Doehlemann G. 2013. Compatibility in the Ustilago maydis-Maize Interaction Requires Inhibition of Host Cysteine Proteases by the Fungal Effector Pit2. PLoS Pathogens 9.

Nostadt R, Hilbert M, Nizam S, et al. 2020. A secreted fungal histidine- and alanine-rich protein regulates metal ion homeostasis and oxidative stress. New Phytologist 227, 1174-1188.

Ökmen B, Kemmerich B, Hilbig D, Wemhöner R, Aschenbroich J, Perrar A, Huesgen PF, Schipper K, Doehlemann G. 2018. Dual function of a secreted fungalysin metalloprotease in Ustilago maydis. The New Phytologist 220, 249-261.

Paulus JK, Kourelis J, Ramasubramanian S, et al. 2020. Extracellular proteolytic cascade in tomato activates immune protease Rcr3. Proceedings of the National Academy of Sciences of the United States of America 117, 17409-17417.

Perez-Riverol Y, Csordas A, Bai J, et al. 2019. The PRIDE database and related tools and resources in 2019: improving support for quantification data. Nucleic Acids Research 47, D442D450.

Perotto S, Daghino S, Martino E. 2018. Ericoid mycorrhizal fungi and their genomes: another side to the mycorrhizal symbiosis? New Phytologist 220, 1141-1147.

Plett JM, Daguerre Y, Wittulsky S, et al. 2014. Effector MiSSP7 of the mutualistic fungus Laccaria bicolor stabilizes the Populus JAZ6 protein and represses jasmonic acid (JA) responsive genes. Proceedings of the National Academy of Sciences of the United States of America 111, 8299-8304.

Plett JM, Kemppainen M, Kale SD, Kohler A, Legué V, Brun A, Tyler BM, Pardo AG, Martin F.

775 Current biology: CB 21, 1197-1203.

Rawlings ND, Barrett AJ, Thomas PD, Huang X, Bateman A, Finn RD. 2018. The MEROPS database of proteolytic enzymes, their substrates and inhibitors in 2017 and a comparison with peptidases in the PANTHER database. Nucleic Acids Research 46, D624-D632.

Richau KH, Kaschani F, Verdoes M, Pansuriya TC, Niessen S, Stüber K, Colby T, Overkleeft HS,

781 Like Cysteine Proteases Displays Subfamily-Specific Characteristics1[C][W]. Plant Physiology

782 158, 1583-1599. 
Rooney HCE, Van't Klooster JW, van der Hoorn RAL, Joosten MHAJ, Jones JDG, de Wit PJGM. 2005. Cladosporium Avr2 inhibits tomato Rcr3 protease required for Cf-2-dependent disease resistance. Science (New York, N.Y.) 308, 1783-1786.

Schardl CL, Leuchtmann A, Spiering MJ. 2004. Symbioses of grasses with seedborne fungal endophytes. Annual Review of Plant Biology 55, 315-340.

Schardl CL, Young CA, Hesse U, et al. 2013. Plant-symbiotic fungi as chemical engineers: multigenome analysis of the clavicipitaceae reveals dynamics of alkaloid loci. PLoS genetics $\mathbf{9}$, e1003323.

Schmid J, Day R, Zhang N, et al. 2016. Host Tissue Environment Directs Activities of an Epichloë Endophyte, While It Induces Systemic Hormone and Defense Responses in Its Native Perennial Ryegrass Host. Molecular Plant-Microbe Interactions ${ }^{\circledR}$ 30, 138-149.

Schulze Hüynck J, Kaschani F, van der Linde K, Ziemann S, Müller AN, Colby T, Kaiser M, Misas Villamil JC, Doehlemann G. 2019. Proteases Underground: Analysis of the Maize Root Apoplast Identifies Organ Specific Papain-Like Cysteine Protease Activity. Frontiers in Plant Science 10.

Scott B, Becker Y, Becker M, Cartwright G. 2012. Morphogenesis, Growth, and Development of the Grass Symbiont Epichlöe festucae. In: Pérez-Martín J,, In: Di Pietro A, eds. Morphogenesis and Pathogenicity in Fungi. Berlin, Heidelberg: Springer Berlin Heidelberg, 243-264.

Scott B, Green K, Berry D. 2018. The fine balance between mutualism and antagonism in the Epichloë festucae-grass symbiotic interaction. Current Opinion in Plant Biology 44, 32-38.

Sekhon RS, Saski C, Kumar R, et al. 2019. Integrated Genome-Scale Analysis Identifies Novel Genes and Networks Underlying Senescence in Maize. The Plant Cell 31, 1968-1989. Pseudomonas syringae Reveals a Virulence Factor That Inhibits Tomato Immune Proteases. PLoS Pathogens 12. Apoplastic effectors secreted by two unrelated eukaryotic plant pathogens target the tomato defense protease Rcr3. Proceedings of the National Academy of Sciences of the United States of America 106, 1654-1659.

815 Plant Science 8.

Stamatakis A. 2014. RAxML version 8: a tool for phylogenetic analysis and post-analysis of large phylogenies. Bioinformatics (Oxford, England) 30, 1312-1313.

818 Takemoto D, Kamakura S, Saikia S, Becker Y, Wrenn R, Tanaka A, Sumimoto H, Scott B. 2011. 
820 complex. Proceedings of the National Academy of Sciences of the United States of America 108, $8212861-2866$.

822 Takemoto D, Tanaka A, Scott B. 2006. A p67Phox-like regulator is recruited to control hyphal

823 branching in a fungal-grass mutualistic symbiosis. The Plant Cell 18, 2807-2821.

824 Tan YY, Spiering MJ, Scott V, Lane GA, Christensen MJ, Schmid J. 2001. In Planta Regulation of 825 Extension of an Endophytic Fungus and Maintenance of High Metabolic Rates in Its Mycelium in 826 the Absence of Apical Extension. Appl. Environ. Microbiol. 67, 5377-5383.

827 Tanaka A, Cartwright GM, Saikia S, Kayano Y, Takemoto D, Kato M, Tsuge T, Scott B. 2013.

828 ProA, a transcriptional regulator of fungal fruiting body development, regulates leaf hyphal

829 network development in the Epichloë festucae-Lolium perenne symbiosis. Molecular

830 Microbiology 90, 551-568.

831 Tanaka A, Christensen MJ, Takemoto D, Park P, Scott B. 2006. Reactive oxygen species play a 832 role in regulating a fungus-perennial ryegrass mutualistic interaction. The Plant Cell 18, 10528331066.

834 Tanaka A, Takemoto D, Hyon G-S, Park P, Scott B. 2008. NoxA activation by the small GTPase 835 RacA is required to maintain a mutualistic symbiotic association between Epichloë festucae and 836 perennial ryegrass. Molecular Microbiology 68, 1165-1178.

837 Thomas EL, van der Hoorn RAL. 2018. Ten Prominent Host Proteases in Plant-Pathogen 838 Interactions. International Journal of Molecular Sciences 19.

839 Tyanova S, Temu T, Cox J. 2016. The MaxQuant computational platform for mass spectrometry 840 - based shotgun proteomics. Nature Protocols 11, 2301-2319.

841 Velasco-Arroyo B, Martinez M, Diaz I, Diaz-Mendoza M. 2018. Differential response of 842 silencing Hvlcy2 barley plants against Magnaporthe oryzae infection and light deprivation. BMC 843 plant biology $18,337$.

844 Wawra S, Fesel P, Widmer H, et al. 2016. The fungal-specific $\beta$-glucan-binding lectin FGB1 845 alters cell-wall composition and suppresses glucan-triggered immunity in plants. Nature 846 Communications 7, 13188.

847 Weber E, Engler C, Gruetzner R, Werner S, Marillonnet S. 2011. A Modular Cloning System for 848 Standardized Assembly of Multigene Constructs. PLoS ONE 6.

849 Young CA, Bryant MK, Christensen MJ, Tapper BA, Bryan GT, Scott B. 2005. Molecular cloning 850 and genetic analysis of a symbiosis-expressed gene cluster for lolitrem biosynthesis from a 851 mutualistic endophyte of perennial ryegrass. Molecular genetics and genomics: MGG 274, 1385229. 
bioRxiv preprint doi: https://doi.org/10.1101/2020 11.06.371211. this version posted November 8,2020 . The copyright holder for this preprint (which was not certified by peer review) is the author/funder, who has granted bioRxiv a license to display the preprint in perpetuity. It is made available under aCC-BY-NC-ND 4.0 International license.

853 Zamioudis C, Pieterse CMJ. 2012. Modulation of host immunity by beneficial microbes.

854 Molecular plant-microbe interactions: MPMI 25, 139-150.

855 Ziemann S, van der Linde K, Lahrmann U, et al. 2018. An apoplastic peptide activates salicylic 856 acid signalling in maize. Nature Plants 4, 172-180. 
857 Table 1. Identified apoplastic inhibitors from plant origin.

858 Shotgun MS analysis of Fl1, CT and mock isotopically labeled apoplastic proteomes

859 screened for the presence of protease inhibitors.

\begin{tabular}{|llrrr|}
\multicolumn{1}{c|}{ ID } & \multicolumn{1}{c}{ Inhibitor annotation } & $\begin{array}{c}\text { unique } \\
\text { peptide }\end{array}$ & $\begin{array}{c}\text { Sequence } \\
\text { coverage [\%] }\end{array}$ & MQ score \\
\hline R_12141 & Cystatin domain (IPR000010) * & 2 & 12.2 & 22.9 \\
\hline R_196 & Proteinase inhibitor I13 ** & 2 & 54.3 & 3.6 \\
\hline R_2071 & Cystatin domain (IPR000010) * & 2 & 8.9 & 10.1 \\
\hline R_2240 & Proteinase inhibitor I13 ** & 1 & 28.2 & 188.6 \\
\hline R_27228 & Cystatin domain (IPR000010) * & 2 & 15.2 & 4.9 \\
\hline R_28259 & Proteinase inhibitor I12, Bowman-Birk type ** & 2 & 17.1 & 51.9 \\
\hline R_386 & Bowman-Birk type proteinase inhibitor (IPR035995) ** & 1 & 10.0 & 25.0 \\
\hline
\end{tabular}

860 Inhibitor identity (ID).

861 Inhibitor annotation based on the uniprot database (www.uniprot.org).

862 MaxQuant score (MQ score).

863 * Cysteine protease inhibitor family (cystatin domain).

$864^{* *}$ Serine proteinase inhibitor family 
bioRxiv preprint doi: https://doi.org/10.1101/2020.11.06.371211; this version posted November 8, 2020. The copyright holder for this preprint (which was not certified by peer review) is the author/funder, who has granted bioRxiv a license to display the preprint in perpetuity. It is made

A $\quad \mathrm{E}+$ E+ available under aCC-BY-NC-BD 4.0 Interntiethal licensE.+
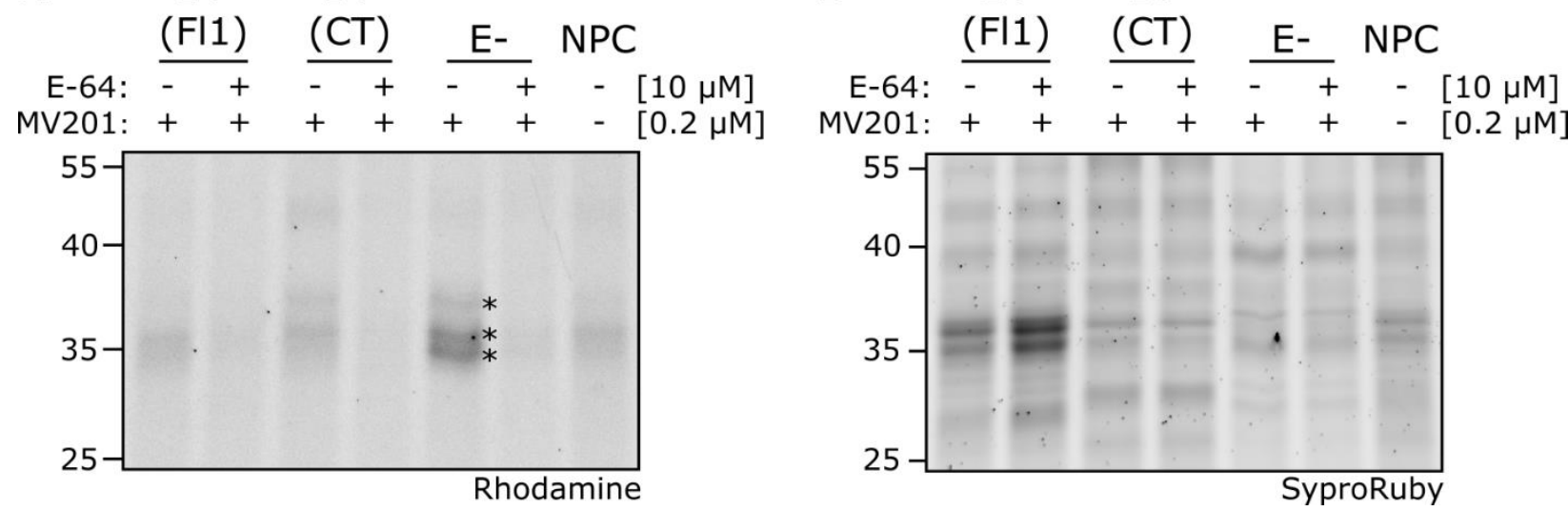

Fig. 1. Activity of apoplastic PLCPs in Epichloe festucae infected Lolium perenne leaves. Leaf apoplastic fluid of endophyte infected (E+) Fl1, CT and mock (E-) plants was isolated. Samples were pre-incubated for 15 min with E-64 or the equivalent amount of DMSO before labelling with the fluorescent activity-based probe MV201. (A) Samples were separated via SDS-PAGE and labelled PLCPs were visualized via in-gel fluorescent scanning using a rhodamine filter (Ex. 532 nm, Em. 580 nm). Asterisks indicate active PLCPs. (B) Sample loading was monitored using SyproRuby staining (Ex. $450 \mathrm{~nm}, \mathrm{Em} .610 \mathrm{~nm}$ ). Numbers on the left side of gel pictures indicate the protein ladder in $\mathrm{KDa}$. 
bioRxiv preprint doi: https://doi.org/10.1101/2020.11.06.371211; this version posted November 8, 2020. The copyright holder for this preprint (which was not certified by peer review) is the author/funder, who has granted bioRxiv a license to display the preprint in perpetuity. It is made

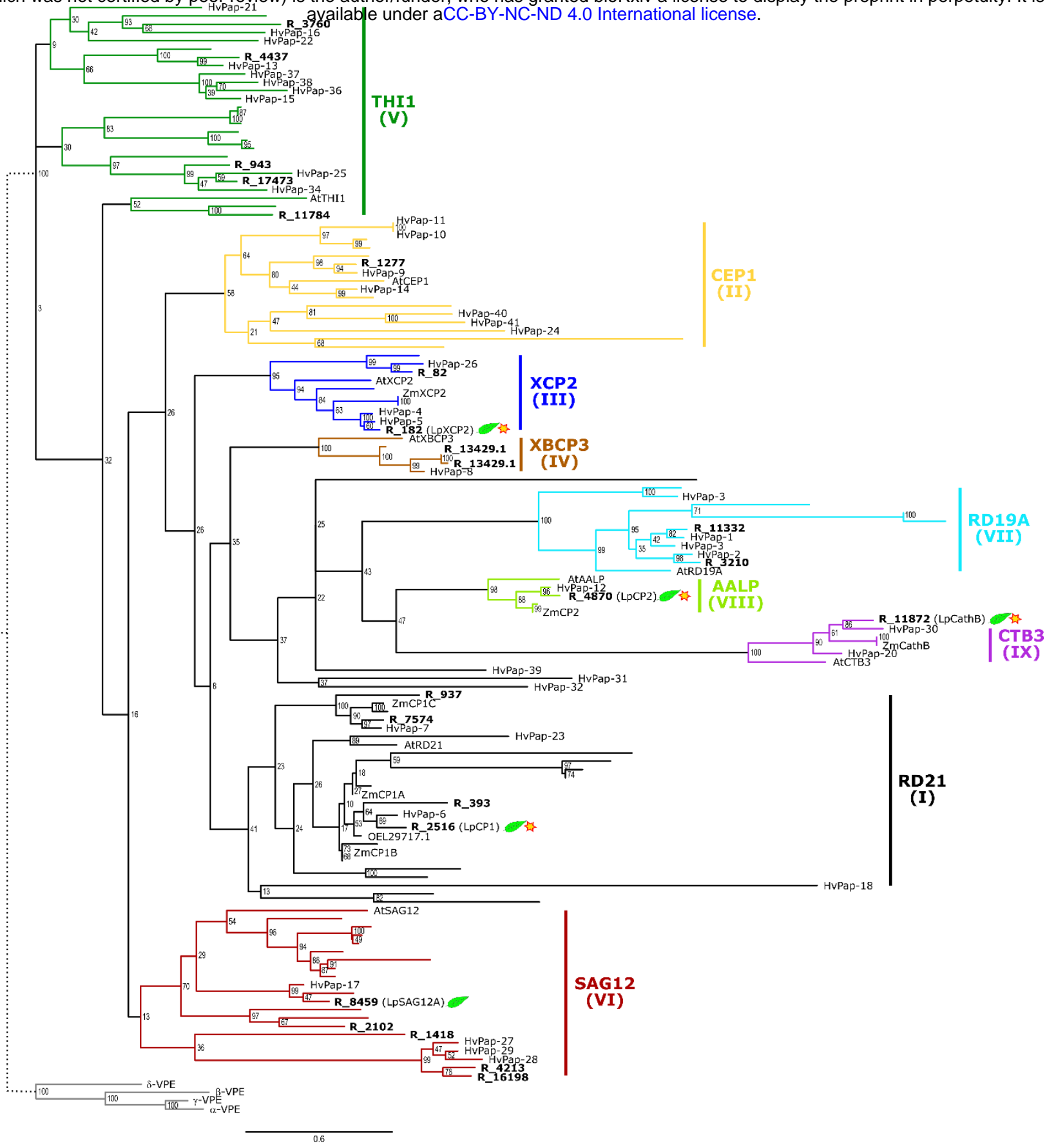

Fig. 2. Phylogeny and subfamily classification of $L$. perenne PLCPs. Phylogenetic analysis was performed using $23 L$. perenne PLCP sequences identified via functional domain analysis ( $R$ numbers), 52 PLCP sequences from the maize line B73 obtained from MEROPS database (www.ebi.ac.uk/merops, branches without label due to space constriction), 6 PLCP sequences from the maize line EGB, and 38 PLCP sequences of Hordeum vulgare (HvPaps). The four legumains (AtVPEs) from $A$. thaliana were used to root the phylogenetic three and one member of each PLCP subfamily also from $A$. thaliana (bold, colored) for the subfamily classification. In this analysis, full length sequences were used, including signal peptide, auto-inhibitory 
bioRxiv preprint doi: https://doi.org/10.1101/2020.11.06.371211; this version posted November 8, 2020. The copyright holder for this preprint (which was not certified by peer review) is the author/funder, who has granted bioRxiv a license to display the preprint in perpetuity. It is made

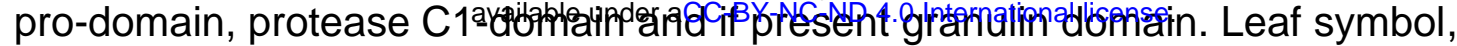
proteases identified in the leaf apoplast proteome analysis; star, proteases found as active enzymes in apoplast using ABPP. To fit the phylogenetic tree in the window the branch of the outgroup has been shortened (dotted line). 
A

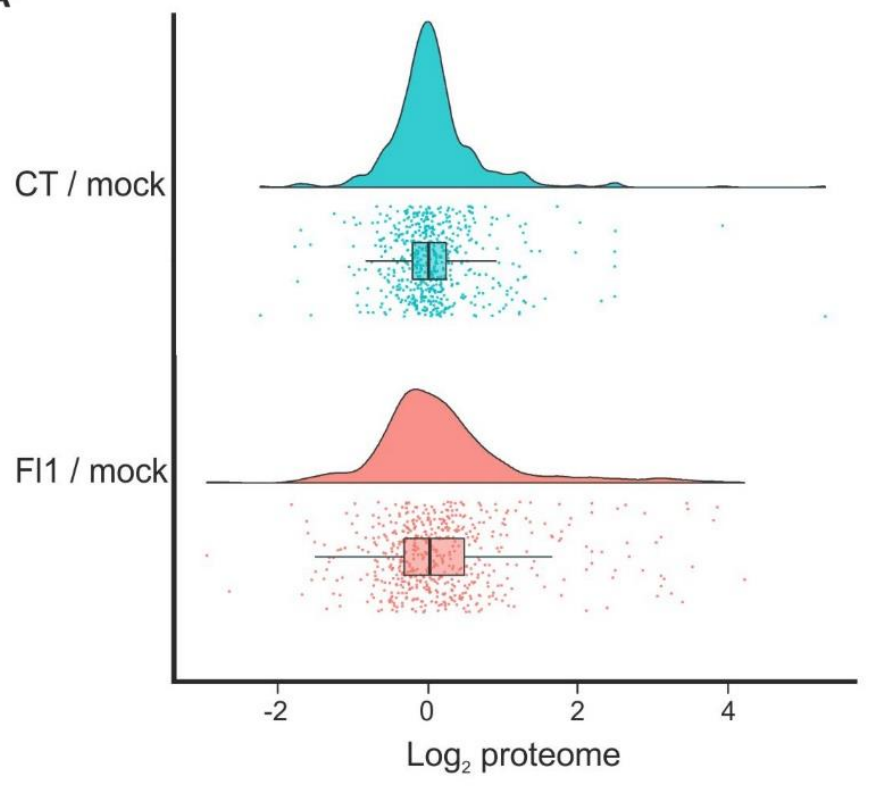

C

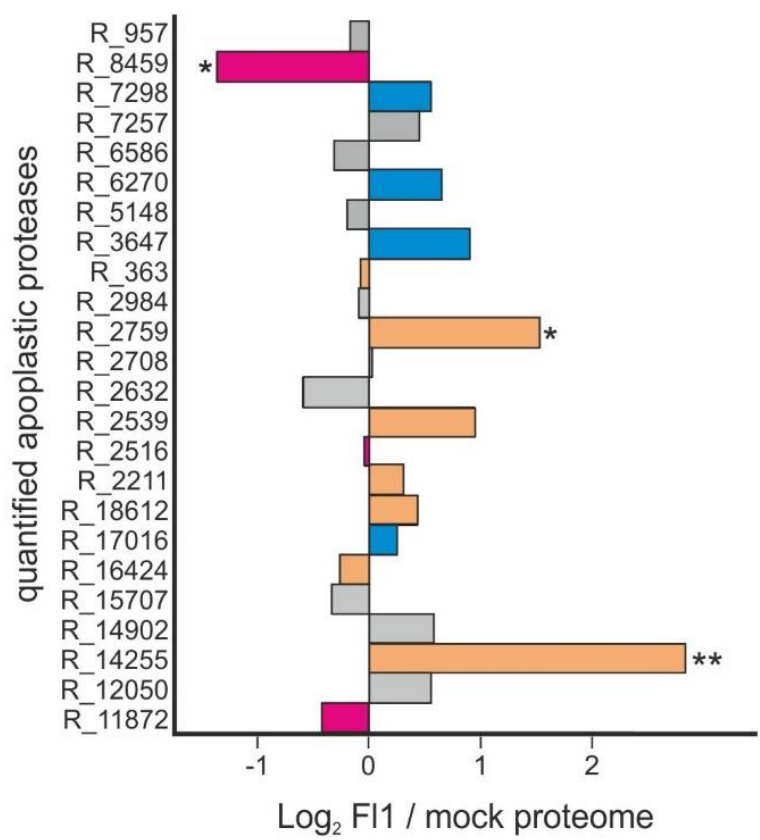

B apoplastic ryegrass proteases $n=51$

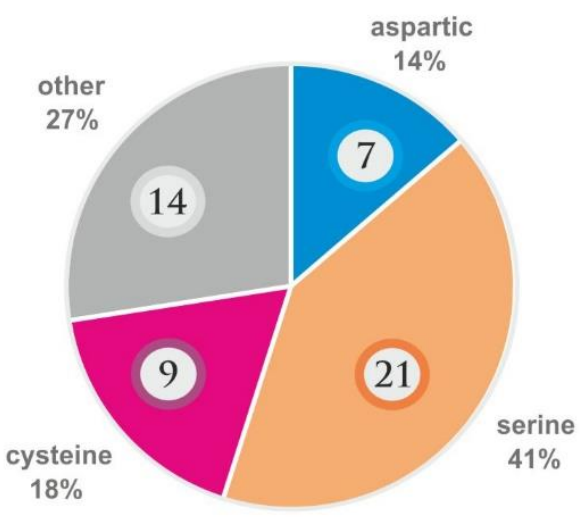

$\square$ other protease

serine protease

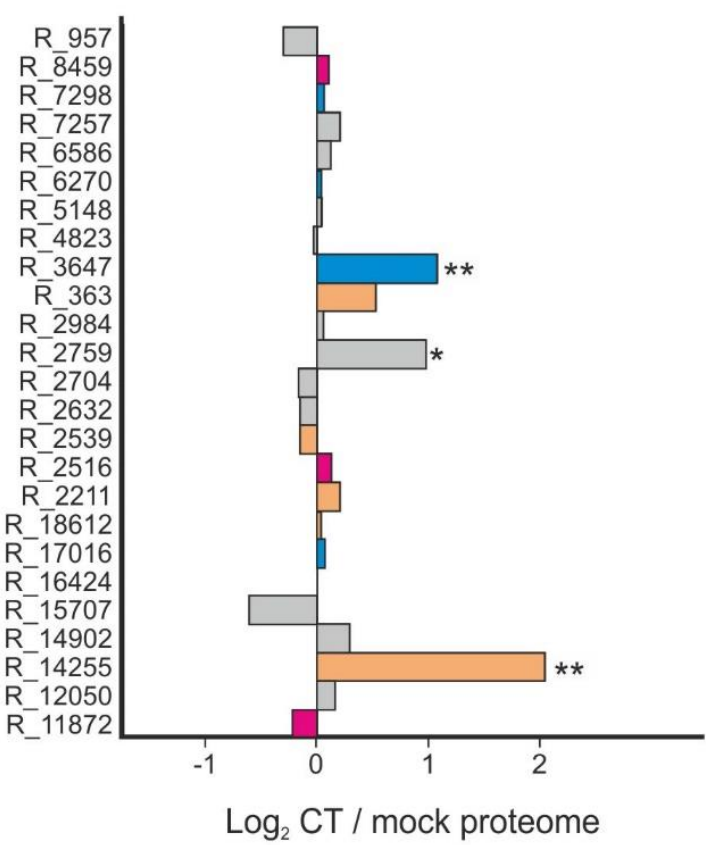

Fig. 3. Apoplast proteome analysis of Fl1 and CT infected and mock-treated leaves. (A) Raincloud plot of $\mathrm{Fl} 1 /$ mock and $\mathrm{CT} /$ mock protein quantification. Apoplast proteins from Fl1, CT and mock-treated plants were stable isotope labelled by reductive dimethylation. The average $\log _{2}$-transformed ratios Fl1/mock and $\mathrm{CT} /$ mock of the 530 and 552 proteins, respectively, were calculated for proteins quantified in at least 
bioRxiv preprint doi: https://doi.org/10.1101/2020.11.06.371211; this version posted November 8, 2020. The copyright holder for this preprint (which was not certified by peer review) is the author/funder, who has granted bioRxiv a license to display the preprint in perpetuity. It is made

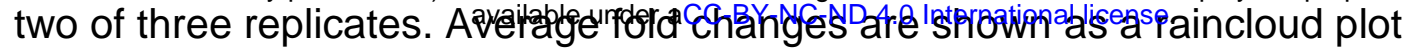
combining a density graph with single dot plots for each quantified protein and a boxplot depicting the mean of the data assembly. (B) Overview of identified apoplastic proteases in the leaf proteome analysis. 51 ryegrass proteases identified in our proteome analysis were grouped according to their catalytic mechanism: aspartic proteases (blue), cysteine proteases (magenta), serine proteases (peach) and other (grey). The total number of each group of identified proteases is shown in circles. The percentage (\%) of protein groups was calculated based on the total number of identified proteases. (C) Quantification of apoplastic proteases in Fl1 and CT infected leaves. Mean $\log _{2}$-transformed ratios $(n=3$ biological replicates, at least quantified in 2 out of 3 replicates) are individually plotted for each of the proteases. 24 and 25 proteases were quantified for FL1/mock and CT / mock, respectively. * represent significant differences $\left({ }^{*}=p<0.05,{ }^{* *}=p<0.01\right.$, LIMMA-moderated Students $t$-test). 
bioRxiv preprint doi: https://doi.org/10.1101/2020.11.06.371211; this version posted November 8, 2020. The copyright holder for this preprint (which was not certified by peer review) is the author/funder, who has granted bioRxiv a license to display the preprint in perpetuity. It is made BaiAgmender aCC-B Unigued 4.0 PHGPational license.

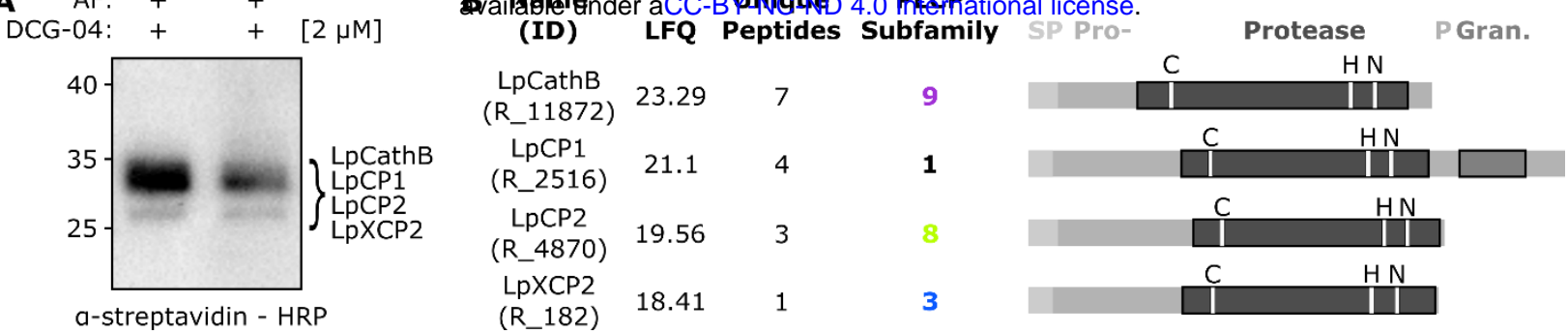

Fig. 4. Identification of active L. perenne apoplastic PLCPs. (A) Pull-down of apoplastic PLCPs using DCG-04. Apoplastic fluid (AF) of endophyte free plants was labelled with the biotinylated probe DCG-04. A background control without DCG-04 (no-probe control, NPC) was performed. Labelled proteins were purified using streptavidin beads. Biotinylated proteins were detected using a-streptavidin-HRP antibody. Shown is a representation of two biological replicates. Estimated sizes for L. perenne (Lp) PLCPs: LpCathB, LpCP1, LpCP2 and LpXCP2 are shown in brackets. (B) Schematic representation of identified apoplastic PLCPs of $L$. perenne. Pull - down samples were subjected to on bead digest (OBD) and subsequent mass spectrometry analysis. Samples were analysed in triplicates and only proteases present in at least two of the three replicates were considered. SP = signal peptide, Pro- $=$ auto-inhibitory prodomain, Protease $=$ protease $\mathrm{C} 1$-domain, $\mathrm{P}=$ Proline-rich domain, Gran = granulin domain. Letters above the protease domain represent the catalytic triad ( $\mathrm{C}$, cysteine; $\mathrm{H}$, histidine; $\mathrm{N}$, asparagine). Coloured numbers show the PLCP subfamily. LFQ, MaxQuant- label-free quantification intensity as a measure of abundance. 
bioRxiv preprint doi: https://doi.org/10.1101/2020.11.06.371211; this version posted November 8, 2020. The copyright holder for this preprint (which was not certified by peer review) is the author/funder, who has granted bioRxiv a license to display the preprint in perpetuity. It is made

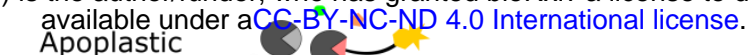

\section{fluids combined}

$(\mathbf{A L})$

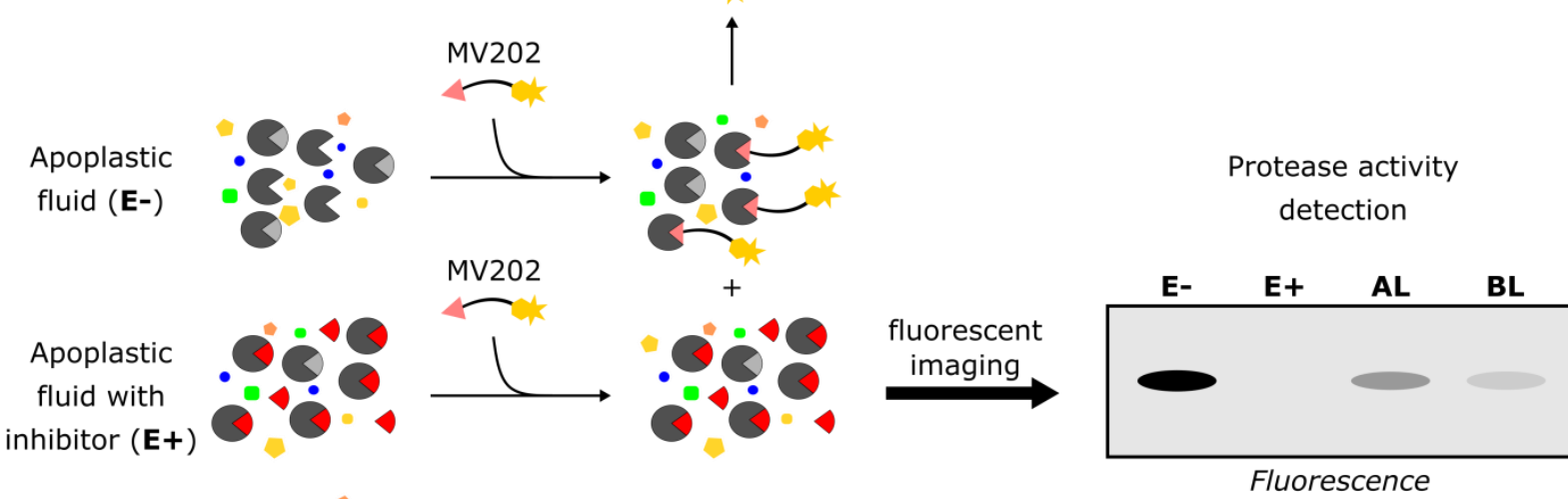

Apoplastic fluids combined

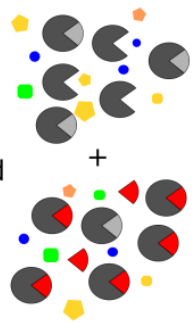

MV202

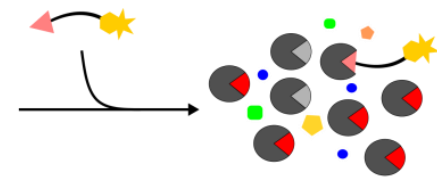

(BL)

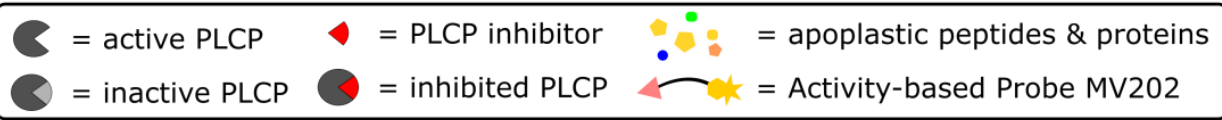

B

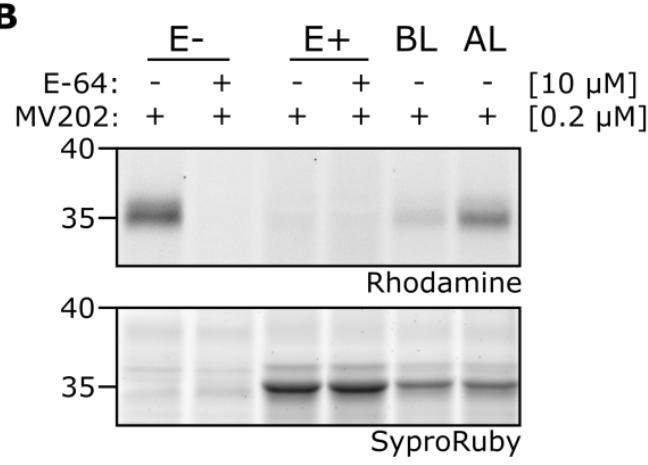

C

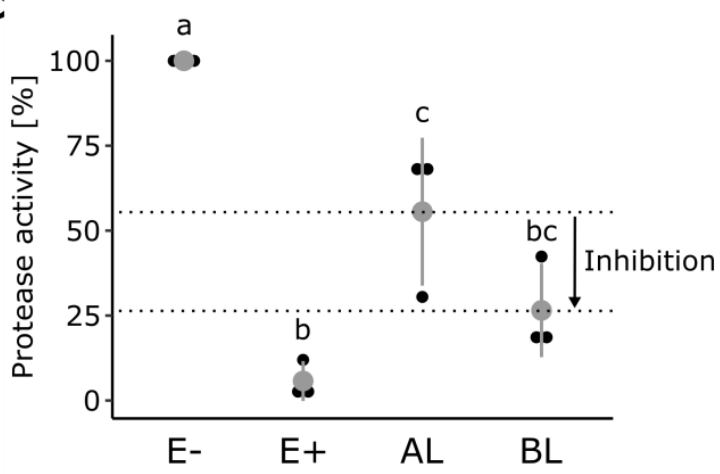

Fig. 5. An apoplastic PLCP inhibitor is present in E. festucae infected plants. (A) Convolution ABPP workflow. Apoplastic fluid with $(\mathrm{E}+)$ and without $(\mathrm{E}-)$ potential PLCP inhibitor is mixed in a 1:1 ratio, allowing the potential PLCP inhibitor in E+ to inhibit active PLCPs in E- before labelling (BL). All three fluids (E-, $\left.E_{+}, B L\right)$, were labelled with the activity-based probe MV202. As control, E+ and E- are mixed (1:1) after labelling ( $A L)$. The fluorescent signals detected in $A L$ should represent the average signals of $\mathrm{E}+$ and $\mathrm{E}$-. If excess inhibitor is present in $\mathrm{E}+$ apoplastic fluid, signal intensities of $B L$ will be lower than signal intensities of $A L$. Figure was adapted from Chandrasekar et al., 2017. (B) An apoplastic PLCP inhibitor is produced during 
bioRxiv preprint doi: https://doi.org/10.1101/2020.11.06.371211; this version posted November 8, 2020. The copyright holder for this preprint (which was not certified by peer review) is the author/funder, who has granted bioRxiv a license to display the preprint in perpetuity. It is made

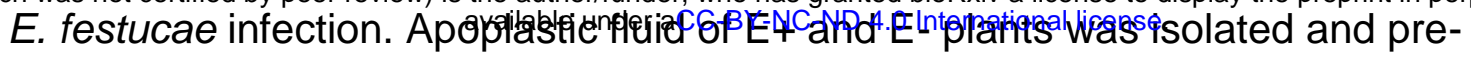
incubated with E-64 or the equivalent amount of DMSO followed by labelling with MV202. One volume of $E+$ fluid and one volume of $E$ - fluid were incubated for 45 min prior to the labelling with MV202 $(\mathrm{BL})$. After labelling $\mathrm{E}+$ and $\mathrm{E}$ - were mixed in a 1:1 ratio (AL). Samples were separated via SDS-PAGE and labelled PLCPs were visualized by in-gel fluorescent scanning using a rhodamine filter. Sample loading was visualized via SyproRuby staining. (C) Quantification of convolution ABPP. Labelling intensities were quantified and normalized to samples treated with E-64. AL and $\mathrm{BL}$ were normalized to the average of $\mathrm{E}-64$ treated samples of $\mathrm{E}+$ and $\mathrm{E}-$. Protease activity of E- was set to $100 \%$ and PLCP activity was calculated in relation to the $\mathrm{E}$ - sample. The red dot represents the mean of three independent biological replicates (black dots), while the red line represents the standard deviation. Different letters indicate significant differences between the means according to Tukey's test $(P<0.05)$. 
bioRxiv preprint doi: https://doi.org/10.1101/2020.11.06.371211; this version posted November 8, 2020. The copyright holder for this preprint (which was not certified by peer review) is the author/funder, who has granted bioRxiv a license to display the preprint in perpetuity. It is made

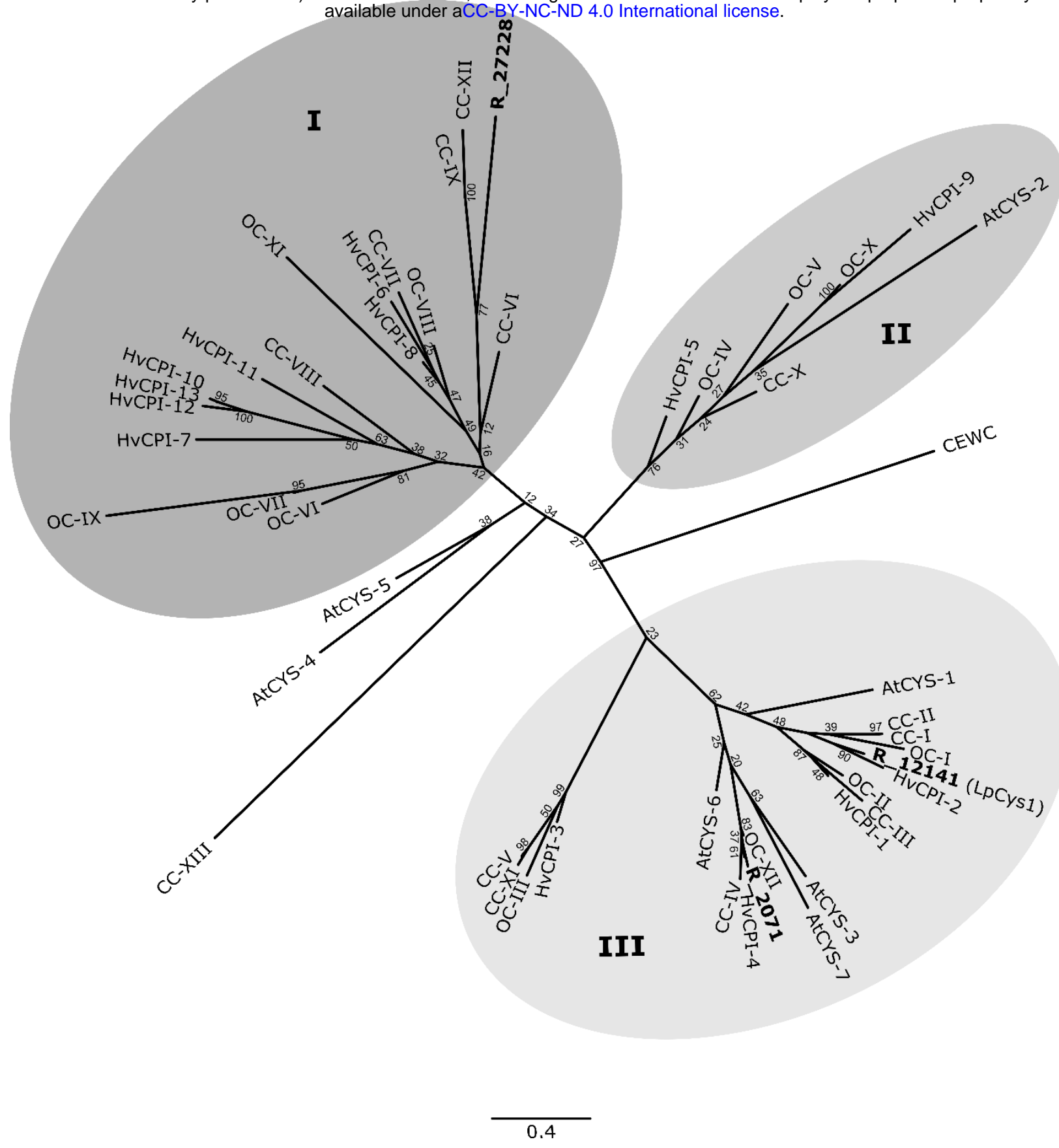

Fig. 6. Phylogentic analysis of identified cystatins from L. perenne. Alignment of fulllength sequences of L. perenne R_12141 (LpCys1), R_2071 and R_27228 (LpCys9) as well as $H$. vulgare ( $\mathrm{HvCPI})$, Z. mays (CC), O. sativa (OC), A. thaliana (AtCYS) and the chicken cystatin (CEWC) were generated using MAFFT. The unrooted radial tree was generated with RAxML (v8.2.12). 100 bootstraps were performed and bootstrap values are indicated. The tree was visualized with FigTree. Grey circles show the main three clusters (I, II and III) obtained for the tested cystatins. 
bioRxiv preprint doi: https://doi.org/10.1101/2020.11.06.371211; this version posted November 8, 2020. The copyright holder for this preprint (which was not certified by peer review) is the author/funder, who has granted bioRxiv a license to display the preprint in perpetuity. It is made

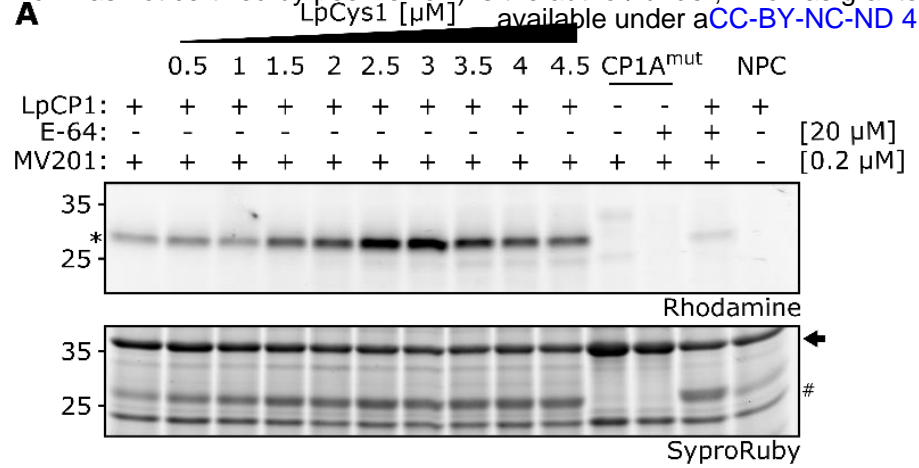

C

LpCys1 $[\mu \mathrm{M}]$

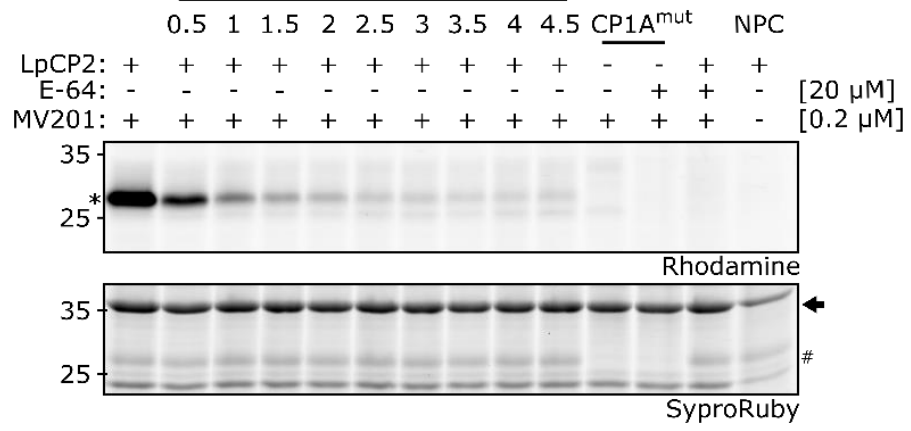

$\mathbf{E}$

LpCys1 $[\mu \mathrm{M}]$

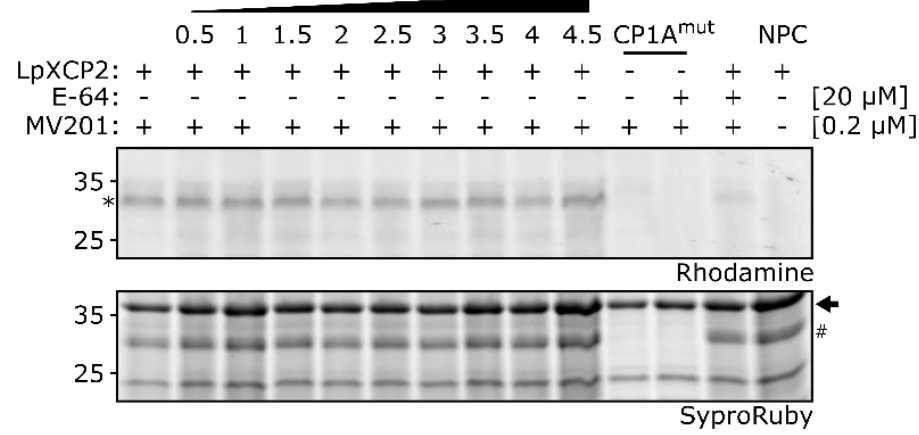

G

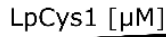

$\begin{array}{lllllllllll}0.5 & 1 & 1.5 & 2 & 2.5 & 3 & 3.5 & 4 & 4.5 & \mathrm{CP} 1 \mathrm{~A}^{\mathrm{mut}} & \mathrm{NPC}\end{array}$

LpCathB: $++++++++++-\overline{-}++$

MV201: $+++++++++++++[0.2 \mu \mathrm{M}]$

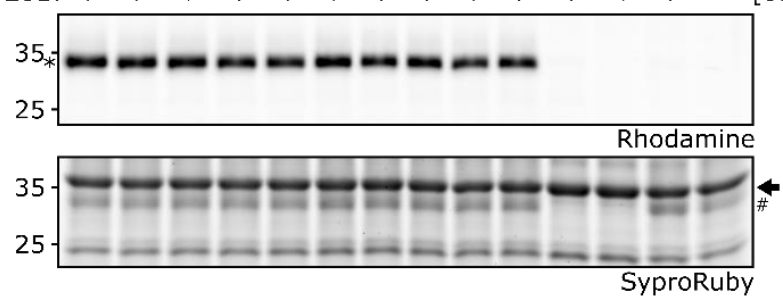

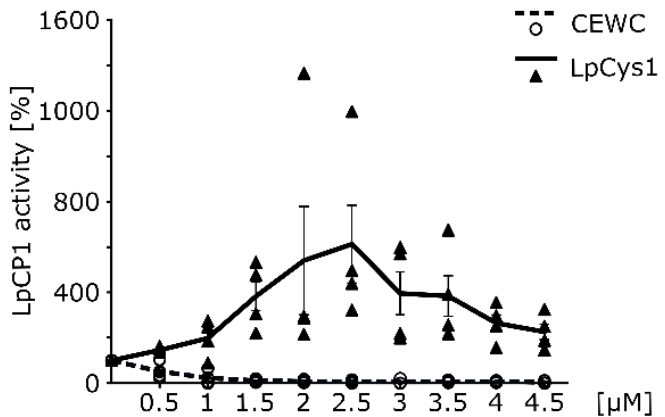

D

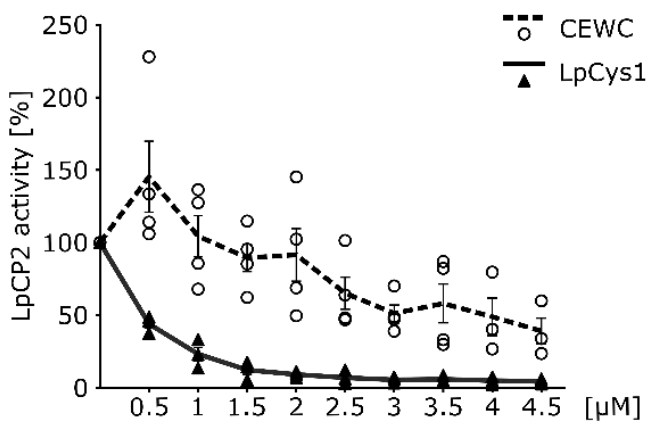

$\mathbf{F}$

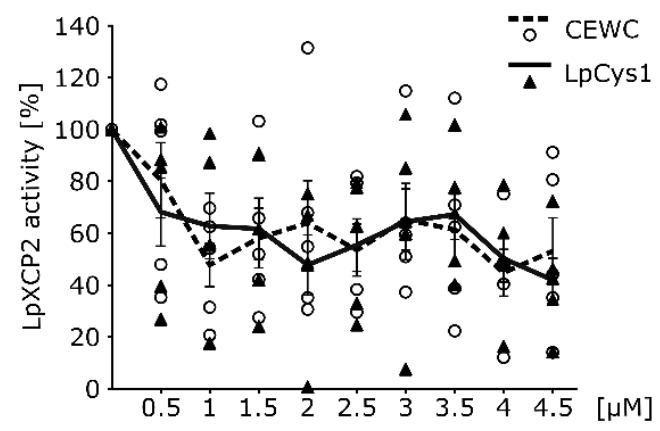

H

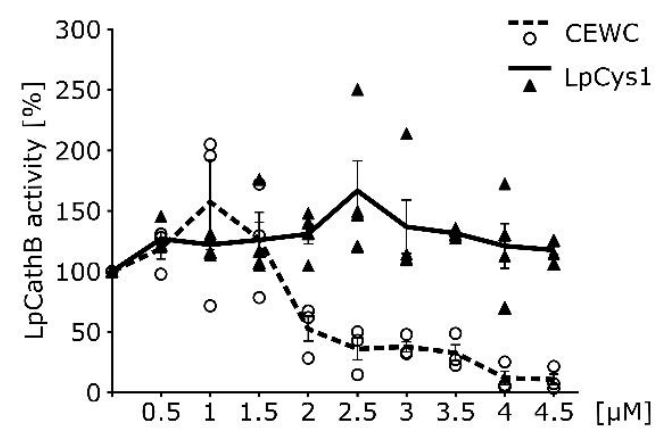

Fig. 7. Inhibitory activity of LpCys1 on overexpressed ryegrass PLCPs. Four PLCPs of $L$. perenne were overexpressed in $N$. benthamiana using Agrobacterium-mediated transformation. Apoplastic fluid containing PLCPs was isolated and monitored using the fluorescent probe MV201. Samples were pre-incubated for 15 min with $20 \mu \mathrm{M} \mathrm{E}$ 64 or a concentration range $(0-4.5 \mu \mathrm{M})$ of heterologous expressed LpCys 1 or 
bioRxiv preprint doi: https://doi.org/10.1101/2020.11.06.371211; this version posted November 8, 2020. The copyright holder for this preprint (which was not certified by peer review) is the author/funder, who has granted bioRxiv a license to display the preprint in perpetuity. It is made

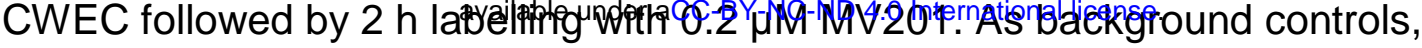
samples containing CP1 $\mathrm{A}^{\text {mut }}$, an inactive maize PLCP, and the no-probe control (NPC) were prepared. The activity and inhibitory effect of LpCys1 on LpCP1 (A), LpCP2 (C), LpXCP2 (E) and LpCathB (G) was analysed using in gel fluorescent scanning and as a loading control SyproRuby staining was performed. For each analysed PLCP (marked as asterisk) signals were quantified and normalized to a loading control signal (marked with an arrow) and activity without inhibitor was set to 100\%. Normalized activity values [\%] for LpCP1 (B), LpCP2 (D), LpXCP2 (F) and LpCathB $(\mathrm{H})$ were plotted against the concentration $[\mu \mathrm{M}]$ of LpCys1 (solid line) and CWEC (dotted line). Error bars represent the standard error of three biological replicates. 